BULLETIN (New Series) OF THE

AMERICAN MATHEMATICAL SOCIETY

Volume 50, Number 3, July 2013, Pages 431-468

S 0273-0979(2013)01415-0

Article electronically published on April 17, 2013

\title{
CHERN-WEIL FORMS AND ABSTRACT HOMOTOPY THEORY
}

\author{
DANIEL S. FREED AND MICHAEL J. HOPKINS \\ In memory of Dan Quillen
}

\begin{abstract}
We prove that Chern-Weil forms are the only natural differential forms associated to a connection on a principal $G$-bundle. We use the homotopy theory of simplicial sheaves on smooth manifolds to formulate the theorem and set up the proof. Other arguments come from classical invariant theory. We identify the Weil algebra as the de Rham complex of a specific simplicial sheaf, and similarly give a new interpretation of the Weil model in equivariant de Rham theory. There is an appendix proving a general theorem about set-theoretic transformations of polynomial functors.
\end{abstract}

\section{Contents}

1. Introduction

2. What is a universal connection?

3. Presheaves and sheaves on manifolds

4. Homotopy theory

5. Simplicial presheaves and weak equivalence

6. Abstract homotopy theory

7. The de Rham complex of $B_{\nabla} G$

8. Proofs

Appendix A. Transformations of polynomial functors 462

About the authors

References

\section{INTRODUCTION}

Invariant theory was studied in the nineteenth century in the context of linear representations of algebraic groups. Given a group $G$ and a linear representation $V$, one seeks polynomials on $V$ which are invariant under the action of $G$. At around the same time Felix Klein formulated his Erlanger Programm [ $\mathrm{K}$ ] which, very roughly, defines geometric concepts as those invariant under a given symmetry group. For example, classical Euclidean geometry studies invariants under the Euclidean group of symmetries of the Euclidean plane $\mathbb{E}^{2}$. The invariants are no longer

Received by the editors January 24, 2013.

2010 Mathematics Subject Classification. Primary 58Axx; Secondary 53C05, 53C08, 55U35.

The work of the first author was supported by the National Science Foundation under grants DMS-0603964, DMS-1207817, and DMS-1160461.

The work of the second author was supported by the National Science Foundation under grants DMS-0906194, DMS-0757293, DMS-1158983. 
polynomials, but may be a numerical invariant of pairs of points (length), of triples of points (the isometry class of a triangle), of a polygon (e.g., the area enclosed), etc. A broader interpretation of Klein's vision formulates Riemannian geometry as the study of invariants of Riemannian manifolds under isometries. Categorical language enables a precise formulation: there is a category whose objects are Riemannian manifolds and whose morphisms are isometries; invariants are functors mapping out of this category, or out of closely related ones.

The problem we investigate here asks for invariants of principal $G$-bundles with connection over smooth manifolds, where $G$ is a fixed Lie group. Specifically, the invariants we seek are differential forms. Long ago Chern and Weil showed that conjugation-invariant polynomials on the Lie algebra $\mathfrak{g}$ define invariant differential forms. Our main result (Theorem 7.20 ) is that these are the only natural differential forms one can construct from a $G$-connection. A similar invariant theory question was crucial in the initial heat equation approach to the Atiyah-Singer index theorem as carried out by Gilkey [G]; see also $\mathrm{ABP}$.

Our focus is not only this specific theorem 1 but also the context we lay out to formulate and prove it. The "invariance" here is under symmetries of $G$-connections as well as smooth maps of manifolds, so we need a framework which tracks both. From another point of view we seek a universal $G$-connection such that any connection on a principal $G$-bundle $P \rightarrow M$ is pulled back from the universal one. Universal connections have been constructed $[\mathrm{NR}, \mathrm{Sch}]$ on infinite-dimensional manifolds (see $[\mathrm{Ku}, \mathrm{R}, \mathrm{DHZ}]$ for further studies) but they have the drawback that classifying maps are not unique. In $\S ₫ 2$ we take the reader on a journey that begins in an elementary way with these traditional universal objects and leads to certain "generalized manifolds": simplicial sheaves on the category of smooth manifolds. We construct a simplicial shea $B_{\nabla} G$ of $G$-connections and a discrete simplicial sheaf $\Omega^{\bullet}$ of differential forms. The precise version of our question becomes a computation: compute all maps $B_{\nabla} G \rightarrow \Omega^{\bullet}$. The actual computation is in $₫ 7$ and $₫ 8$, where we prove (Theorem 7.20) that the classical construction of Chern and Weil captures all differential forms naturally associated to a $G$-connection. Once the framework is set up, the computation involves only ideas from differential geometry and invariant theory: no simplicial sheaves. One piece of the invariant theory - a proof that set-theoretic transformations of polynomial functors are polynomial-may be of independent interest; it is worked out in the appendix.

Our work is a new take on Chern-Weil theory and equivariant de Rham theory. In the world of simplicial sheaves we define the total space $E_{\nabla} G$ of the universal bundle with connection. We prove (Theorem 7.19) that its de Rham complex is the Weil algebra, the star character in H. Cartan's treatment C1, C2 of Chern-Weil theory. Traditionally, the Weil algebra is used as a finite-dimensional model of the infinite dimensional de Rham complex of a Hilbert manifold model of $E G$, or a finite dimensional approximation thereof. Here we offer a geometric interpretation of the Weil algebra as precisely the de Rham complex of $E_{\nabla} G$. We also prove

\footnotetext{
${ }^{1}$ Some version of this theorem may already be known, but we could not find a reference. One novelty may be Lemma 8.6 we do not need to assume that the forms we consider are local functions of the connection-we prove it.

${ }^{2}$ Pronounced " $B$ nabla $G$ ". The idea of considering $E_{\nabla} G \rightarrow B_{\nabla} G$ as a universal principal $G$ bundle with connection surely dates back at least to the early 1970s and to ideas implicit in $[\mathrm{Br} \mathrm{D}]$. We could not find an explicit reference from that era, however. The construction does appear in the much more recent [FSS.
} 
a generalization. For a $G$-manifold $X$ we define a version of the Borel quotient using $E_{\nabla} G$ and prove (Theorem 7.28 ) that the de Rham complex of this simplicial Borel quotient is precisely the Weil model in equivariant de Rham theory.

A crucial ingredient in our discussion is abstract homotopy theory, which we describe in 96 . Were we to only discuss differential forms, even in their incarnation as the sheaf of sets $\Omega^{\bullet}$, there would be no need for homotopy theory. But principal bundles have automorphisms, usually called gauge transformations, and a fixed $G$ connection may be stabilized by a nontrivial subgroup of gauge transformations. So these objects may appear in several equivalent forms. We can describe a $G$-bundle directly as a certain type of fiber bundle $P \rightarrow X$, or alternatively we can specify it by an open cover of the manifold $X$ and transition functions. There are similar alternative descriptions of a $G$-connection. Abstract homotopy theory provides a mechanism for systematically identifying these alternatives.

In recent years abstract homotopy theory has had a profound impact on various parts of algebraic geometry, as well as on low-dimensional topology. Here we use abstract homotopy theory in differential geometry. There are closely related contexts in which abstract homotopy theory also plays a crucial role. For example, generalized differential cohomology groups [HS] are most naturally defined in this world; see $[\mathrm{Bu}$. for a recent exposition. We remark that they generalize Cheeger-Simons cohomology groups [ChS], which in turn refine Chern-Weil theory. Abstract homotopy theory also lies at the foundation of derived differential geometry [Sp, Joy]. In a different direction, simplicial sheaves provide a good framework in which to define a general notion of a "field" in the sense of classical and quantum field theory [FT, Appendix].

We offer this paper as a tribute to Dan Quillen. He introduced abstract homotopy theory in Q1, Q2 , and he also wrote about Chern-Weil theory in Q3, MQ. Dan was an exceptionally clear and elegant mathematical thinker. He leaves behind a legacy of profound and powerful mathematics which will continue to inspire for a very long time.

\section{WHAT IS A UNIVERSAL CONNECTION?}

In this section we motivate the question "What is a universal connection?", for which we begin with some topological analogues. Let $\Sigma$ be a compact surface with no boundary. Its simplest topological invariant is the Euler number $\chi(\Sigma) \in \mathbb{Z}$, defined for a triangulation of $\Sigma$ by Euler's famous formula $\chi(\Sigma)=V-E+F$, where $V$ is the number of vertices, $E$ the number of edges, and $F$ the number of faces. For our purposes we replace this combinatorial definition with one based on a smooth structure. We focus on the tangent bundle $\pi: T \Sigma \rightarrow \Sigma$, the linearization of $\Sigma$ which assigns to each point $p \in \Sigma$ the two-dimensional tangent space $\pi^{-1}(p)=T_{p} \Sigma$. The tangent bundle $T \Sigma$ is the union of these two-dimensional real vector spaces, collected into a smooth manifold. The Euler number measures the "twisting" of the tangent spaces as $p$ varies over $\Sigma$. One qualitative indication that there is twisting for $\Sigma=S^{2}$ is the hairy ball theorem, which states that there is no smooth nonzero vector field on the 2-sphere: every hairy sphere has a bald spot! If there were no twisting, then we could identify each tangent space with the standard twodimensional real vector space $\mathbb{R}^{2}$, and then promote a nonzero vector at a single point to a nonzero global vector field. 
To obtain a quantitative measurement of the twisting of $T \Sigma \rightarrow \Sigma$, we ask: What is the maximally twisted smooth family of two-dimensional real vector spaces? One source of twisted smooth families are Grassmannians: any real vector space $W$ determines the Grassmannian $G r_{2}(W)$ of 2-planes in $W$, named after the nineteenth century German mathematician and high school teacher Hermann Grassmann. The disjoint union of the 2-planes is the total space of a vector bundle $\pi: E(W) \rightarrow G r_{2}(W)$. It turns out that every two-dimensional real vector bundle is pulled back from this one as long as we take $W$ to be infinite dimensional. Therefore, our quest for a universal real vector bundle of rank two takes us outside the world of finite-dimensional manifolds. Furthermore, even if we allow infinitedimensional manifolds, the classifying manifold is not unique. For example, we can take $W=\mathbb{R}^{\infty}$ with a direct limit topology and similarly topologize $G r_{2}\left(\mathbb{R}^{\infty}\right)$. Or we can take $W$ to be an infinite-dimensional real Hilbert space and correspondingly construct a Hilbert manifold $G r_{2}(W)$. There is no canonical choice for the classifying space. Rather, there is a theorem in topology that any two choices are homotopy equivalent. We return to this issue in 4 . For now we observe that the cohomology of a universal parametrizing space $G r_{2}(W)$ is independent of the choice, and for any choice we locate a universal Euler class $\chi \in H^{2}\left(G r_{2}(W)\right) 3$ Then the universality expresses the tangent bundle to $\Sigma$ as a pullback

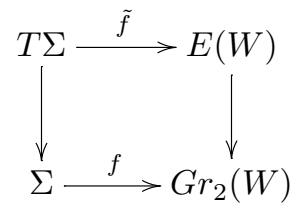

and the Euler number of $\Sigma$ is the value of $f^{*} \chi$ on the fundamental class of $\Sigma$ - the "integral" of $f^{*} \chi$ over $\Sigma$. (The pullback property means that $\tilde{f}$ is an isomorphism from $T_{p} \Sigma$ to the vector space labeled by $f(p)$, for each $p \in \Sigma$.) Not only is $G r_{2}(W)$ not unique, but the classifying map $f$ is also not unique, though any two are homotopic.

The Euler class of a 2-plane bundle is the first example of a characteristic class [MS. More generally, for any $\sqrt{4}$ Lie group $G$ we consider a principal $G$-bundle $\pi: P \rightarrow M$ over a smooth manifold $M$. By definition $G$ acts freely on the manifold $P$ with quotient map $\pi$, and the action admits local slices. The principal bundle associated to $T \Sigma \rightarrow \Sigma$ has $G=G L_{2} \mathbb{R}$, and the total space is the set of isomorphisms $\mathbb{R}^{2} \rightarrow T_{p} \Sigma$ for all $p \in \Sigma$. There are again infinite-dimensional universal bundles $E G \rightarrow B G$, unique up to homotopy, and elements of $H^{\bullet}(B G)$ are universal topological invariants of principal $G$-bundles. Characteristic classes are the solution to a two-step problem: find a universal $G$-bundle and compute its cohomology.

The problem we consider in this paper is to construct "differential geometric characteristic classes". To motivate it, let us return to our smooth surface $\Sigma$ and now suppose it is endowed with a Riemannian metric $g$. The differential geometers of the eighteenth and nineteenth centuries studied the concrete case of a surface $\Sigma \subset \mathbb{E}^{3}$ embedded in Euclidean 3-space with the induced metric. Befitting the local nature of the metric, we now ask not for global measurements of topological twisting, but

\footnotetext{
${ }^{3}$ More precisely, the universal Euler class lies in cohomology twisted by a canonical local system constructed from the orientations of the two-planes in $W$.

${ }^{4}$ Throughout we assume $G$ has finitely many components.
} 
rather for local measurements of geometric twisting. Gauss's Theorema Egregium provides a single function $K_{g}: \Sigma \rightarrow \mathbb{R}$, the Gauss curvature, which is an invariant of the metric, and it measures the deviation from flatness. We remark that there is also a canonical measure $d A_{g}$ constructed from the metric - from lengths and angles, we compute areas - and the Gauss-Bonnet theorem asserts that the integral $\frac{1}{2 \pi} \int_{\Sigma} K_{g} d A_{g}$ equals the Euler number $\chi(\Sigma)$.

The curvature is a combination of second derivatives of the metric. The Italian school in the late nineteenth century constructed a new geometric object constructed from first derivatives, the Levi-Civita connection. In the twentieth century connections were recognized to have independent interest, and at mid-century Charles Ehresmann E] formulated the notion of a connection $\Theta$ on a principal $G$ bundle $P \rightarrow M$. Let $\mathfrak{g}$ denote the Lie algebra of $G$. A connection on $P$ is a 1 -form $\Theta \in \Omega^{1}(P ; \mathfrak{g})$ which satisfies two conditions: (i) the restriction of $\Theta$ to each fiber is the Maurer-Cartan form $\theta \in \Omega^{1}(G ; \mathfrak{g})$; and (ii) if $R_{g}: P \rightarrow P$ denotes the action of $g \in G$, then $R_{g}^{*} \Theta=\operatorname{Ad}_{g^{-1}} \Theta$. Our problem is to construct local invariants of connections. A natural home for these invariants is the generalization of functions: differential forms. Recall that for any manifold $M$ differential forms $\Omega^{\bullet}(M)$ have a differential $d$ which defines the de Rham complex

$$
\Omega^{0}(M) \stackrel{d}{\longrightarrow} \Omega^{1}(M) \stackrel{d}{\longrightarrow} \Omega^{2}(M) \stackrel{d}{\longrightarrow} \cdots .
$$

We are led, then, to the following two-step problem:

Problem 2.3. Construct a universal $G$-connection on a principal $G$-bundle $E_{\nabla} G \rightarrow B_{\nabla} G$.

Problem 2.4. Compute the de Rham complex of $B_{\nabla} G$.

The idea of the universal connection is that any connection $\Theta$ on a bundle $P \rightarrow M$ is pulled back from a map $M \rightarrow B_{\nabla} G$, analogously to (2.1). Once we seek a universal $G$-connection, it is natural to seek a universal de Rham complex as well.

Problem 2.5. Construct a universal space of differential forms $\Omega^{\bullet}$ and a universal de Rham complex.

If we in addition impose uniqueness of the classifying map of a differential form, then we seek some object $\Omega^{\bullet}$ such that for any smooth manifold $M$ the space of maps $M \rightarrow \Omega^{\bullet}$ is

$$
\operatorname{Map}\left(M, \Omega^{\bullet}\right)=\Omega^{\bullet}(M) .
$$

Just as classifying spaces of 2-plane bundles and of principal $G$-bundles are not finite-dimensional manifolds, but rather are infinite-dimensional manifolds, so too we construct $\Omega^{\bullet}$ and $B_{\nabla} G$ as "generalized manifolds". We introduce that generalization and solve Problem 2.5 in the next section. By contrast $B_{\nabla} G$ is not as rigid as $\Omega^{\bullet}$, hence we defer its construction, which requires homotopy theory, to $\$ 5$. The solution to Problem 2.4 is stated as Theorem 7.20 and proved in $₫ 8$.

\section{Presheaves And Sheaves on MANifolds}

Let Man denote the category whose objects are smooth finite-dimensional manifolds and whose morphisms are smooth maps between manifolds. The righthand side of (2.6) is a set attached to each smooth manifold $M$. Furthermore, 
if $f: M^{\prime} \rightarrow M$ is a smooth map, then there is a pullback map of differential forms

$$
\Omega^{\bullet}\left(M^{\prime}\right) \stackrel{f^{*}}{\longleftarrow} \Omega^{\bullet}(M),
$$

and the pullback of the composition of two maps is the composition of the pullbacks. Let Set denote the category of sets. We summarize the structural properties of differential forms by the statement that

$$
\begin{aligned}
\Omega^{\bullet}: \mathbf{M a n}^{\mathrm{op}} & \longrightarrow \text { Set } \\
M & \longmapsto \Omega^{\bullet}(M)
\end{aligned}
$$

is a functor. Here op denotes the opposite category in which all arrows are reversed: differential forms pull back.

Definition 3.3. A presheaf on manifolds is a functor Man $^{\mathrm{op}} \rightarrow$ Set.

In this context we view $M$ as a "test manifold" on which we evaluate the presheaf. The presheaf itself is to be considered as a new geometric object which generalizes a manifold. To justify that point of view, we must first see that manifolds may be regarded as presheaves. Let $X$ be a smooth finite-dimensional manifold, and define the associated presheaf $\mathcal{F}_{X}$

$$
\begin{aligned}
\mathcal{F}_{X}: \operatorname{Man}^{\mathrm{op}} & \longrightarrow \text { Set } \\
M & \longmapsto \operatorname{Man}(M, X) .
\end{aligned}
$$

To a test manifold $M$ this presheaf assigns the set of all smooth maps $M \rightarrow X$, the set of maps from $M$ to $X$ in the category Man. Throughout we use standard constructions and notations in categories; for example ' $C(X, Y)$ ' is used for the set of morphisms $X \rightarrow Y$ in the category $C$.

Remark 3.5. The notion of a presheaf is more familiar over a fixed manifold $X$. A presheaf over $X$ assigns a set to each open set in $X$ and there are coherent restriction maps, so it may be viewed as a functor

$$
\operatorname{open}(X)^{\text {op }} \longrightarrow \text { Set }
$$

on the category whose objects are open subsets of $X$ and whose morphisms are inclusions of open sets. A good general reference on presheaves and sheaves is [MM].

If presheaves on manifolds are meant to generalize manifolds, then we must be able to do geometry with presheaves, and to begin we define maps between presheaves, so a category Pre of presheaves.

Definition 3.7. Let $\mathcal{F}^{\prime}, \mathcal{F}$ be presheaves on manifolds. Then a map $\varphi: \mathcal{F}^{\prime} \rightarrow \mathcal{F}$ is a natural transformation of functors. Thus for each test manifold $M$ there is a map $\mathcal{F}^{\prime}(M) \stackrel{\varphi(M)}{\longrightarrow} \mathcal{F}(M)$ of sets such that for every smooth map $M^{\prime} \stackrel{f}{\rightarrow} M$ of test manifolds, the diagram

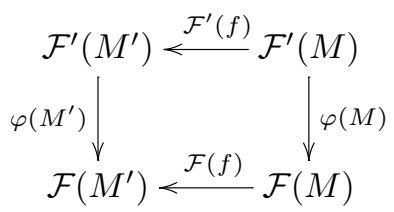

commutes. 
This definition has the nice feature that if the domain presheaf $\mathcal{F}^{\prime}$ is that of a smooth manifold $X$, as in (3.4), then we use $M=X$ as a test manifold and so determine $\varphi: \mathcal{F}_{X} \rightarrow \mathcal{F}$ by its value on $\operatorname{id}_{X} \in \mathcal{F}_{X}(X)$, which is an element $\varphi\left(\operatorname{id}_{X}\right)$ of the set $\mathcal{F}(X)$. More formally, we have the following.

Lemma 3.9 (Yoneda). For any presheaf $\mathcal{F}$, evaluation on $X$ determines an isomorphism $\operatorname{Pre}\left(\mathcal{F}_{X}, \mathcal{F}\right) \cong \mathcal{F}(X)$.

Here 'Pre $\left(\mathcal{F}_{X}, \mathcal{F}\right)$ ' denotes the set of maps in the category of presheaves introduced in Definition 3.7. Because of Lemma 3.9 for any presheaf $\mathcal{F}$ we sometimes write an element of $\mathcal{F}(X)$ as a map $X \rightarrow \mathcal{F}$.

Remark 3.10. It is important to observe that smoothness is encoded in the presheaf $\mathcal{F}_{X}$, even though the values of $\mathcal{F}_{X}$ are sets with no additional structure. For example, a special case of Lemma 3.9 is that for any smooth manifolds $X, Y$

$$
\operatorname{Pre}\left(\mathcal{F}_{X}, \mathcal{F}_{Y}\right) \cong \mathcal{F}_{Y}(X)=\operatorname{Man}(X, Y) .
$$

In other words, maps $\mathcal{F}_{X} \rightarrow \mathcal{F}_{Y}$ of presheaves are precisely smooth maps $X \rightarrow Y$ of manifolds.

Remark 3.12. What appears in (3.11) are discrete sets, but the construction actually remembers much more. For if $S$ is any smooth manifold, then the set of smooth maps from $S$ into the function space of maps $X \rightarrow Y$ is $\operatorname{Man}(S \times X, Y)$; see Example 3.29 below.

Remark 3.13. The map $X \mapsto \mathcal{F}_{X}$ defines a functor from Man into the category of presheaves on manifolds. Then (3.11) asserts that this functor induces an isomorphism on Hom-sets, i.e., is "fully faithful". So Man is a full subcategory of presheaves that expresses precisely the sense in which presheaves are generalized manifolds.

Another consequence of Lemma 3.9 is that for any smooth manifold $X$, we have

$$
\operatorname{Pre}\left(\mathcal{F}_{X}, \Omega^{\bullet}\right) \cong \Omega^{\bullet}(X) .
$$

Of course, the definition (3.2) is rigged to make this true. What is more, in the world of presheaves on manifolds, we can define differential forms on the presheaf $\Omega^{q}$ for each $q \in \mathbb{Z}^{\geq 0}$. For example, there is a canonical $q$-form

$$
\boldsymbol{\omega}^{q}=\operatorname{id}_{\Omega^{q}}: \Omega^{q} \longrightarrow \Omega^{q},
$$

which to every test manifold $M$ assigns the identity map on $\Omega^{q}(M)$. The form $\boldsymbol{\omega}^{q}$ enjoys a tautological uniqueness property: if $\omega \in \Omega^{q}(X)$, then there is a unique map $\varphi: \mathcal{F}_{X} \rightarrow \Omega^{q}$ such that $\varphi^{*}\left(\boldsymbol{\omega}^{q}\right)=\omega$. The map $\varphi$ is defined by $\varphi(f)=f^{*} \omega$ for $f: M \rightarrow X$. It is now straightforward to write the universal de Rham complex

$$
\Omega^{0} \stackrel{d}{\longrightarrow} \Omega^{1} \stackrel{d}{\longrightarrow} \Omega^{2} \stackrel{d}{\longrightarrow} \Omega^{3} \stackrel{d}{\longrightarrow} \cdots,
$$

which on a test manifold $M$ is the de Rham complex (2.2) on $M$. The complex (3.16) is the solution to Problem 2.5. Again, we emphasize that we have constructed a universal object into which there are unique classifying maps.

The reader may feel that we have defined away the problem with no gain. To dispel such illusions we retort that any presheaf on manifolds has a de Rham complex, as illustrated here. 
Theorem 3.17. The de Rham complex of $\Omega^{1}$ is isomorphic to

$$
\mathbb{R} \stackrel{0}{\longrightarrow} \mathbb{R} \stackrel{1}{\longrightarrow} \mathbb{R} \stackrel{0}{\longrightarrow} \mathbb{R} \stackrel{1}{\longrightarrow} \cdots .
$$

In particular, the de Rham cohomology of $\Omega^{1}$ is

$$
H_{d R}^{\bullet}\left(\Omega^{1}\right) \cong \begin{cases}\mathbb{R}, & \bullet=0 \\ 0, & \bullet \neq 0\end{cases}
$$

The vector space $\Omega^{q}\left(\Omega^{1}\right)$ has dimension one and is generated by $\left(d \boldsymbol{\omega}^{1}\right)^{\wedge(q / 2)}$ if $q$ is even and $\boldsymbol{\omega}^{1} \wedge\left(d \boldsymbol{\omega}^{1}\right)^{\wedge(q-1) / 2}$ if $q$ is odd, where $\boldsymbol{\omega}^{1}$ is defined in (3.15). This is a special case 5 of Theorem 7.19, whose proof appears in 8 . It does not appear that this special case has a substantially simpler proof than the general case.

Remark 3.20. Theorem 3.17 unpacks into a concrete statement in "invariant theory". Namely, $\tau \in \Omega^{q}\left(\Omega^{1}\right)=\operatorname{Pre}\left(\Omega^{1}, \Omega^{q}\right)$ is a natural construction of a $q$-form from a 1-form. Thus if $M$ is a smooth manifold and $\omega \in \Omega^{1}(M)$, we have $\tau(\omega) \in \Omega^{q}(M)$, and for any smooth map $f: M^{\prime} \rightarrow M$ we have $\tau\left(f^{*} \omega\right)=f^{*} \tau(\omega)$. The language of presheaves encodes this naturality statement, and it opens the way to more intricate definitions and theorems.

Remark 3.21. Theorem 3.17 shows that the generalized manifold $\Omega^{1}$ has a rather simple de Rham complex, e.g., it is finite dimensional in each degree. By contrast, the space of 1 -forms on a positive-dimensional ordinary manifold $X$ is infinite dimensional.

The examples of presheaves we have encountered are determined by local data: functions and forms are determined by their values on arbitrarily small open sets. We abstract that property, which is not satisfied by a general presheaf.

Definition 3.22. Let $\mathcal{F}: \operatorname{Man}^{\text {op }} \rightarrow$ Set be a presheaf. Then $\mathcal{F}$ is a sheaf if for every manifold $M$ and every open cover $\left\{U_{\alpha}\right\}$ of $M$

$$
\mathcal{F}(M) \longrightarrow \prod_{\alpha_{0}} \mathcal{F}\left(U_{\alpha_{0}}\right) \longrightarrow \prod_{\alpha_{0}, \alpha_{1}} \mathcal{F}\left(U_{\alpha_{0}} \cap U_{\alpha_{1}}\right)
$$

is an equalizer diagram.

This is the usual gluing property of a sheaf: given $x_{\alpha_{0}} \in \mathcal{F}\left(U_{\alpha_{0}}\right)$ such that the restrictions of $x_{\alpha_{0}}$ and $x_{\alpha_{1}}$ to $U_{\alpha_{0}} \cap U_{\alpha_{1}}$ agree, there is a unique $x \in \mathcal{F}(M)$ which restricts on $U_{\alpha_{0}}$ to $x_{\alpha_{0}}$. Functions satisfy this gluing property, and more generally $\Omega^{\bullet}$ is a sheaf. We often say $\mathcal{F}(U)$ is the set of sections of the sheaf $\mathcal{F}$ on the open set $U$.

Each presheaf $\mathcal{F}$ has a universal map $\mathcal{F} \rightarrow \mathbf{a} \mathcal{F}$ to a sheaf $\mathbf{a} \mathcal{F}$, called the sheafification of $\mathcal{F}$. Universality means that if $\mathcal{F}^{\prime}$ is a sheaf and $\mathcal{F} \rightarrow \mathcal{F}^{\prime}$ is a map (of presheaves), then there is a unique sheaf map a $\mathcal{F} \rightarrow \mathcal{F}^{\prime}$ making the diagram

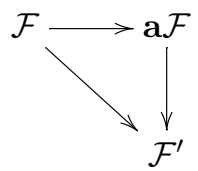

\footnotetext{
${ }^{5}$ in which the Lie group is $G=\mathbb{T}$, the circle group
} 
commute. In the language of categories this is expressed by saying that the forgetful functor Sh $\longrightarrow$ Pre from the category of sheaves to the category of presheaves has a left adjoint $\mathbf{a}$. The functor $\mathbf{a}$ is the associated sheafification functor.

Just as presheaves on a fixed topological space have stalks, so too do presheaves on manifolds. As all manifolds are locally diffeomorphic to a ball in affine space, we may as well record only one stalk in each dimension.

Definition 3.25. Let $\mathcal{F}: \operatorname{Man}^{\text {op }} \rightarrow$ Set be a presheaf. For $m \in \mathbb{Z}^{\geq 0}$ the $m$ dimensional stalk of $\mathcal{F}$ is the colimit

$$
\underset{r \rightarrow 0}{\operatorname{colim}} \mathcal{F}\left(B^{m}(r)\right),
$$

where $B^{m}(r) \subset \mathbb{A}^{m}$ is the ball of radius $r$ about the origin in $m$-dimensional affine space.

If $r^{\prime}<r$, there is an inclusion $B^{m}\left(r^{\prime}\right) \hookrightarrow B^{m}(r)$, and so a restriction map $\mathcal{F}\left(B^{m}(r)\right)$ $\rightarrow \mathcal{F}\left(B^{m}\left(r^{\prime}\right)\right)$. The colimit is explicitly computed by taking the disjoint union of all $\mathcal{F}\left(B^{m}(r)\right)$ and identifying $x \in \mathcal{F}\left(B^{m}(r)\right)$ and $x^{\prime} \in \mathcal{F}\left(B^{m}\left(r^{\prime}\right)\right)$ if $x$ maps to $x^{\prime}$ under restriction. The stalks are sets with no additional structure. For example, the $m$-dimensional stalk of the sheaf $\Omega^{\bullet}$ is the set of germs of smooth differential forms at the origin in $\mathbb{A}^{m}$.

Remark 3.27. The information contained in a sheaf is essentially the collection of stalks, one for each nonnegative integer $m$, and the maps between them induced from germs of smooth maps between affine spaces.

Remark 3.28. The map $\mathcal{F} \rightarrow \mathbf{a} \mathcal{F}$ induces an isomorphism on stalks.

Function spaces provide another class of examples of sheaves on manifolds.

Example 3.29 (Function spaces). Let $X, Y$ be smooth manifolds. The space of smooth maps $X \rightarrow Y$ may be given the structure of an infinite-dimensional Fréchet manifold, but we can alternatively work with it as a sheaf $\mathcal{F}$. Namely, for a test manifold $M$ let $\mathcal{F}(M)$ be the set of smooth maps $M \times X \rightarrow Y$. There are many variations. For example, if we replace $Y$ by the sheaf $\Omega^{1}$, then $\mathcal{F}(M)=\Omega^{1}(M \times X)$. Notice that by promoting 1 -forms on $X$ to a sheaf, we attach to a test manifold $M$ 1-forms on the product $M \times X$, not partial 1-forms defined only on tangent vectors pointing along $X$.

A first attack on Problem 2.3 might begin by considering the presheaf $\mathcal{F}$ which to a test manifold $M$ assigns

$$
\mathcal{F}(M)=\{\text { isomorphism classes of } G \text {-connections on } M\}
$$

for a fixed Lie group $G$. Let $\mathfrak{g}$ be the Lie algebra of $G$. An element of $\mathcal{F}(M)$ is an equivalence class of principal $G$-bundles $P \rightarrow M$ with connection $\Theta \in$ $\Omega^{1}(P ; \mathfrak{g})$, where two connections $\Theta, \Theta^{\prime}$ are equivalent if there is a bundle isomorphism $\varphi: P^{\prime} \rightarrow P$ covering the identity map on $M$ such that $\varphi^{*}(\Theta)=\Theta^{\prime}$. It is standard to verify that $\mathcal{F}$ is a presheaf on manifolds. By contrast, $\mathcal{F}$ is not a sheaf. For consider $M=S^{1}$ with the open cover $U_{1}, U_{2} \subset S^{1}$ by the complements of two distinct points. Then $U_{1} \cap U_{2}$ is diffeomorphic to two disjoint intervals and (3.23) 
reduces to the diagram
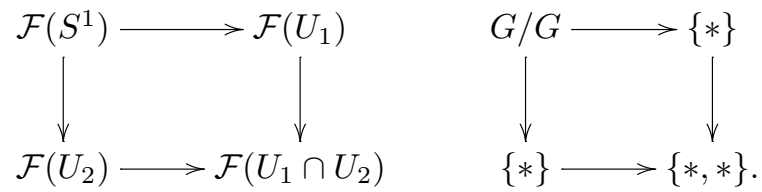

A $G$-connection on $S^{1}$ is determined up to isomorphism by the conjugacy class of its holonomy, from which $\mathcal{F}\left(S^{1}\right)=G / G$ is the set of conjugacy classes. (Here $G$ acts on itself by conjugation.) Any connection on an interval is isomorphic to the trivial connection. Since (3.31) fails to be a pullback diagram, $\mathcal{F}$ is not a sheaf.

Isomorphism classes of connections do not glue since connections have automorphisms. In the preceding example trivial connections on the intervals $U_{1}, U_{2}$ can glue to a nontrivial connection on $S^{1}$, and this explains the failure of $\mathcal{F}$ to be a sheaf. In the next section we explore techniques for tracking the automorphisms and so ultimately for gluing connections.

\section{Homotopy theоRY}

In this section we come to grips with the following question.

Problem 4.1. What mathematical structure $\mathcal{S}$ describes the collection of $G$ connections on a fixed manifold $M$ ?

Let us first take the simplest case $G=\mathbb{Z} / 2 \mathbb{Z}$, the cyclic group of order two. A principal $\mathbb{Z} / 2 \mathbb{Z}$-bundle over $M$ is simply a double cover $P \rightarrow M$, and it has a unique connection. So a $\mathbb{Z} / 2 \mathbb{Z}$-connection is a double cover. Problem 4.1 specializes to: What mathematical structure $\mathcal{S}$ encodes all double covers of $M$ ? Notice that the fiber of a double cover consists of two points, which we can think of as the two points of norm one in a real one-dimensional vector space equipped with an inner product. Since the space of inner products is contractible - any two are related by a positive scalar - double covers are "topologically equivalent" to real line bundles. Thus we are returning to the classification problem at the beginning of $\$ 2$, only now for real line bundles in place of real 2-plane bundles. Consider, then, the Grassmannian $G r_{1}(W)$ of lines in any real vector space $W$, also called the projective space $\mathbb{P}(W)$. For $W$ infinite dimensional it is a good model for the collection of all lines, and we might be tempted to take the space of smooth maps $M \rightarrow G r_{1}(W)$ as the answer to Problem 4.1. But as in 2 there are different ways to make $G r_{1}(W)$ an infinite-dimensional manifold, and for none of them is there a 1:1 correspondence between maps $M \rightarrow G r_{1}(W)$ and real line bundles over $M$. So we seek a different approach in which classifying maps are unique.

The nonuniqueness of classifying maps is due to the fact that a double cover $P \rightarrow$ $M$ has internal symmetries, namely maps $\varphi: P \rightarrow P$ which cover the identity map on $M$. If $M$ is connected there is a unique nonidentity symmetry, the deck transformation which flips the sheets of the double cover. We need a mathematical structure which tracks the symmetries and, more generally, tracks isomorphisms $\varphi: P^{\prime} \rightarrow P$ between different double covers. One possibility is to organize double covers and their isomorphisms into a groupoid.

Definition 4.2. A groupoid $\mathcal{G}$ is a category in which every arrow is invertible. Two groupoids $\mathcal{G}, \mathcal{G}^{\prime}$ are equivalent if they are equivalent categories, i.e., if there exist functors $f: \mathcal{G} \rightarrow \mathcal{G}^{\prime}, g: \mathcal{G}^{\prime} \rightarrow \mathcal{G}$ and natural equivalences $g \circ f \simeq \operatorname{id}_{\mathcal{G}}, f \circ g \simeq \operatorname{id}_{\mathcal{G}^{\prime}}$. 
We write $\mathcal{G}=\left\{\mathcal{G}_{0}, \mathcal{G}_{1}\right\}$, where $\mathcal{G}_{0}$ is the collection of objects and $\mathcal{G}_{1}$ the collection of morphisms. A functor $f: \mathcal{G} \rightarrow \mathcal{G}^{\prime}$ is an equivalence if and only if it is essentially surjective and fully faithful. The first condition means that for each $x^{\prime} \in \mathcal{G}_{0}^{\prime}$ there exists $x \in \mathcal{G}_{0}$ and $\left(f x \rightarrow x^{\prime}\right) \in \mathcal{G}_{1}^{\prime}$. The second means that for all $x, y \in \mathcal{G}_{0}$ the map $f: \mathcal{G}(x, y) \rightarrow \mathcal{G}^{\prime}(f x, f y)$ is a bijection, where $\mathcal{G}(x, y)$ is the set of arrows in $\mathcal{G}$ from $x$ to $y$.

The following criterion will be useful later. A groupoid $\mathcal{G}$ is discrete if for all $x, y \in \mathcal{G}_{0}$ the set $\mathcal{G}(x, y)$ is either empty or contains a unique element.

Lemma 4.3. Let $\mathcal{G}, \mathcal{G}^{\prime}$ be discrete groupoids. Then if $f: \mathcal{G} \rightarrow \mathcal{G}^{\prime}$ is surjective on objects, it is an equivalence of groupoids.

A set may be regarded as a groupoid with only identity arrows, which in particular is a discrete groupoid. Conversely, every discrete groupoid is equivalent to a set.

Returning to double covers, let

$$
\mathcal{G}_{0}=\mathcal{G}_{0}(M)=\text { collection of double covers } \pi: P \rightarrow M ;
$$

$$
\mathcal{G}_{1}=\mathcal{G}_{1}(M)=\text { collection of commutative diagrams }
$$

Isomorphisms of double covers, which comprise $\mathcal{G}_{1}$, are part of the structure.

As an example consider $M=S^{1}$. The groupoid (4.4) is very large; each double cover of the circle is a distinct element of $\mathcal{G}_{0}\left(S^{1}\right)$. But up to isomorphism there are only two distinct double covers of the circle. One is the trivial double cover $\pi_{0}: S^{1} \times \mathbb{Z} / 2 \mathbb{Z} \rightarrow S^{1}$. Choose a particular nontrivial double cover $\pi_{1}$ : identify $S^{1} \subset \mathbb{C}$ as the set of complex numbers of unit norm, and let $\pi_{1}$ be the squaring map. Let $\mathcal{G}^{\prime}$ be the groupoid with $\mathcal{G}_{0}^{\prime}=\left\{\pi_{0}, \pi_{1}\right\}$ and $\mathcal{G}_{1}^{\prime}=\left\{\operatorname{id}_{\pi_{0}}, \operatorname{id}_{\pi_{1}}, \varphi_{0}, \varphi_{1}\right\}$. (Here $\varphi_{i}$ is the deck transformation of $\pi_{i}$.) There is an inclusion map $g: \mathcal{G}^{\prime} \rightarrow \mathcal{G}$, and we claim it is an equivalence of groupoids. To construct a functor $f: \mathcal{G} \rightarrow \mathcal{G}^{\prime}$, for each double cover $\pi: P \rightarrow S^{1}$ choose an isomorphism $\pi \rightarrow \pi_{i}$ for $i=0$ or $i=1$ according to whether $\pi$ is trivializable or not. The functor $f$ maps $\pi$ to $\pi_{i}$, and under the chosen isomorphisms any arrow $\pi \rightarrow \pi^{\prime}$ in $\mathcal{G}$ maps to either $\operatorname{id}_{\pi_{i}}$ or $\varphi_{i}$. The composition $f \circ g$ is the identity functor on $\mathcal{G}^{\prime}$, and the chosen isomorphisms are the data of a natural equivalence from $g \circ f$ to id $\mathrm{G}_{\mathcal{G}}$.

Returning to Problem 4.1, it is not enough to simply give a mathematical structure $\mathcal{S}$. We must also discuss a notion of equivalence $\simeq$ between two instances of $\mathcal{S}$, a relation we term weak equivalence. This is a key point: the solution to Problem 4.1 is a pair $(\mathcal{S}, \simeq)$. Now $\mathcal{S}=$ Groupoid is a venerable mathematical structure, and with the notion of equivalence in Definition 4.2 it is a valid solution to Problem 4.1, For what we do in this paper it is sufficient, and we will use it to good advantage, but nonetheless we describe a more general solution which applies more broadly. As one motivation, most of us are much fonder of the geometric notion $\mathcal{S}=$ Space with weak equivalences defined to be (weak) homotopy equivalences. There is a direct relationship of groupoids and spaces. There is a functor

$$
\text { Groupoid } \longrightarrow \text { Space }
$$

which assigns a classifying space to each groupoid; see Definition 4.21 below. 
Theorem 4.6 ([S], Proposition 2.1). Equivalent groupoids map to homotopy equivalent spaces.

If we apply (4.5) to the groupoid $\mathcal{G}(\mathrm{pt})$ of double covers of a point, then we obtain a space homotopy equivalent to the projective space $\mathbb{R} \mathbb{P}^{\infty}=\mathbb{P}\left(\mathbb{R}^{\infty}\right)=G r_{1}\left(\mathbb{R}^{\infty}\right)$ of the infinite-dimensional real vector space $W=\mathbb{R}^{\infty}$ with the direct limit topology.

Remark 4.7. For $M=$ pt there is a very efficient groupoid $\mathcal{G}^{\prime \prime}$ weakly equivalent to $\mathcal{G}(\mathrm{pt})$ with $\mathcal{G}_{0}^{\prime \prime}$ a set with a single element and $\mathcal{G}_{1}^{\prime \prime}$ the cyclic group of order two.

Our solution to Problem 4.1 is a mathematical structure Set $_{\Delta}$ called simplicial set, which sits between groupoid and space: there are functors

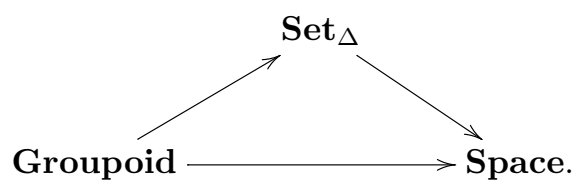

We begin with a formal definition, which also explains the notation 'Set ${ }_{\Delta}$ '. Let $\Delta$ be the category whose objects are nonempty totally ordered finite sets and whose morphisms are order-preserving maps. It is equivalent to a category with one object for each nonnegative integer.

Definition 4.9. A simplicial set is a functor $F: \Delta^{\text {op }} \rightarrow$ Set. A map $F_{\bullet}^{\prime} \rightarrow F_{\bullet}$ of simplicial sets is a natural transformation of functors. Simplicial sets form a category $\operatorname{Set}_{\Delta} 6$

If $F_{\bullet}$ is a simplicial set we define the sequence of sets $F_{0}, F_{1}, F_{2}, \ldots$ by $F_{n}=$ $F(\{0,1,2, \ldots, n\})$, whence the bullet subscript in the notation ' $F_{\bullet}$ '. Intuitively, $F_{n}$ is the collection of $n$-simplices of the simplicial set $F_{\bullet}$. The order-preserving maps between the canonical totally ordered sets $\{0,1,2, \ldots, n\}$ give the diagram

$$
F_{0} \lesseqgtr-\gtrless F_{1} \underset{\longleftarrow-\geqslant}{\longleftarrow} F_{2} \cdots .
$$

The left solid arrows are the $n+1$ face maps of an $n$-simplex. The right dashed arrows are the $n$ degeneracy maps of an $n$-simplex. The composition laws in $\Delta$ induce relations among the face and degeneracy maps. We proceed directly to some illustrative examples, and we recommend $[\mathrm{MP}, \mathrm{Fr}$ for expository accounts and $[\mathrm{Cu}, \mathrm{Ma}, \mathrm{GJ}]$.

Example 4.11 (Groupoids as simplicial sets). Let $\mathcal{G}=\left\{\mathcal{G}_{0}, \mathcal{G}_{1}\right\}$ be a groupoid. The associated simplicial set $F(\mathcal{G})$ • has $F(\mathcal{G})_{0}=\mathcal{G}_{0}$ and $F(\mathcal{G})_{1}=\mathcal{G}_{1}$. In other words, the 0-simplices of $F(\mathcal{G})$ • are the objects of the groupoid and the 1-simplices are the arrows. For $n>1$ define $F(\mathcal{G})_{n}$ to be the collection of compositions of $n$ arrows in $\mathcal{G}_{1}$. The two face maps $F(\mathcal{G})_{0} \longleftarrow F(\mathcal{G})_{1}$ are the source and target maps of the groupoid, and the degeneracy map $F(\mathcal{G})_{0}-->F(\mathcal{G})_{1}$ assigns the identity arrow $\mathrm{id}_{\pi}$ to each object $\pi \in \mathcal{G}_{0}$. There is an elegant formal definition of $F(\mathcal{G})$. An object $S \in \Delta$ is a category whose objects are the elements of $S$ and there is a unique morphism $s \rightarrow s^{\prime}$ if $s \leq s^{\prime}$ in $S$. Then the value of $F(\mathcal{G}): \Delta^{\text {op }} \rightarrow$ Set on $S$ is the collection of functors $S \rightarrow \mathcal{G}$. Since the $n$-simplices for $n>1$ are determined

\footnotetext{
${ }^{6}$ The notation derives from that in topology, where $B^{A}$ is the set of maps $A \rightarrow B$; lowering the domain in 'Set ${ }_{\Delta}$ ' to a subscript "dualizes" $\Delta$ to the opposite category.
} 
by the 0 - and 1-simplices, the simplicial set determined by a groupoid only carries information about topology in dimensions zero and one.

Example 4.12 (Discrete simplicial sets). If $S$ is any set, we promote it to a groupoid $\mathcal{G}$ with $\mathcal{G}_{0}=\mathcal{G}_{1}=S$; there are only identity arrows. By Example4.11 this determines a simplicial set $S$. with $S_{n}=S$ for all $n$; all simplices of positive degree are degenerate. We usually omit the lower bullet in the notation for a constant simplicial set. If $T$ is a topological space, we can form the discrete simplicial set of the underlying point set of $T$. A discrete simplicial set only encodes topology in dimension zero. Discrete simplicial sets are also called constant simplicial sets.

We can remember more of the topology of a space $T$ by the singular simplicial set.

Example 4.13 (Spaces as simplicial sets). A space $T$ determines a simplicial set Sing. $T$ defined by $\operatorname{Sing}_{n} T=\operatorname{Space}\left(\Delta^{n}, T\right)$, the set of continuous maps of the standard $n$-simplex $\Delta^{n}$ into $T$. The face and degeneracy maps are induced by the corresponding maps of standard simplices. The simplicial set Sing. $T$ encodes topology in all dimensions.

Example 4.14 (Group actions). Let $S$ be a set and $G$ a group which acts on $S$. There is a groupoid $\mathcal{G}_{\bullet}$ which describes this group action. Namely, the set of objects is $\mathcal{G}_{0}=S$ and the set of arrows is $\mathcal{G}_{1}=G \times S$ : for every $s \in S$ and $g \in G$ there is an arrow with source $s \in \mathcal{G}_{0}$ and target $g \cdot s \in \mathcal{G}_{0}$. The group action defines the composition of arrows. The corresponding simplicial set is

$$
S \underset{\gtrless}{\longleftarrow} G \times S \underset{\longleftarrow-\geqslant}{\longleftarrow} G \times G \times S \cdots
$$

Example 4.16. Let $X$ be a smooth manifold and $\mathcal{U}=\left\{U_{\alpha}\right\}_{\alpha \in A}$ an open cover. There is an associated simplicial set $F(\mathcal{U})$ • which starts off as

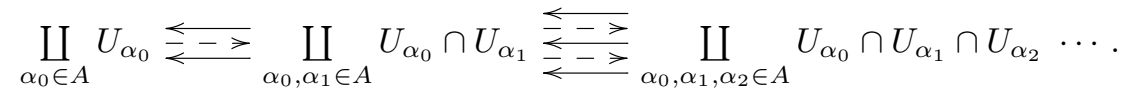

There a natural map $f: F(\mathcal{U}) \bullet \rightarrow X$ • to the discrete simplicial set $X_{\bullet}$ defined at each level by inclusion. The simplicial set $F(\mathcal{U}) \bullet$ is derived as in Example 4.11 from a groupoid $\mathcal{G}$, where $\mathcal{G}_{i}=F(\mathcal{U})_{i}, i=0,1$. The inclusion map $f$ is an equivalence of groupoids, as follows immediately from Lemma 4.3 .

Definition 4.18. Let $F_{\bullet}$ be a simplicial set. Then the set $\pi_{0}\left(F_{\bullet}\right)$ is defined as the coequalizer of

$$
F_{0} \longleftarrow F_{1} .
$$

For example, if $F_{\bullet}$ is the simplicial set associated to the groupoid in Example 4.14, then $\pi_{0}\left(F_{\bullet}\right)$ is the set of orbits of the $G$-action on $S$.

The functor Set $_{\Delta} \rightarrow$ Space in (4.8) is called geometric realization. We briefly recall the definition Mi]. Define the standard $n$-simplex

$$
\Delta^{n}=\left\{\left(t^{0}, t^{1}, \ldots, t^{n}\right) \in \mathbb{A}^{n+1}: t^{i} \geq 0, t^{0}+\cdots+t^{n}=1\right\} .
$$

If $I \in \Delta$ is any nonempty ordered finite set, then it has a unique isomorphism to $0,1, \ldots, n$ for some $n$, and we define $\Sigma(I)=\Delta^{n}$. There is an easy extension to a functor $\Sigma: \Delta \rightarrow$ Space. 
Definition 4.21. Let $F: \Delta^{\mathrm{op}} \rightarrow$ Set be a simplicial set. The geometric realization $\left|F_{\bullet}\right|$ is the quotient space of the disjoint union

$$
\coprod_{I} \Sigma(I) \times F(I)
$$

by the identifications $\left(\theta_{*} t, x\right) \sim\left(t, \theta^{*} x\right)$ for all morphisms $\theta$ in $\Delta$.

More concretely, one can replace (4.22) by a disjoint union of $\Delta^{n} \times F_{n}$ and the maps in (4.10) give the gluings. In this description $F_{n}$ parametrizes the set of $n$-simplices in $\left|F_{\bullet}\right|$; the face and degeneracy maps tell how to glue them together.

Example 4.23. The geometric realization of the discrete simplicial set built from a set $S$ (Example 4.12) is canonically isomorphic to $S$. The geometric realization of the simplicial set $F(\mathcal{U})$. associated to a cover of a manifold $X$ is homotopy equivalent to $X$ with the discrete topology; this follows from the remarks at the end of Example 4.16 and Theorem 4.6. More interesting are the geometric realizations in Example 4.13 and Example 4.14 the geometric realization of the simplicial set Sing. $T$ associated to a space $T$ is homotopy equivalent to $T$ (with its given topology), and the geometric realization of the groupoid built from a discrete group $G$ acting on a set $S$ is homotopy equivalent to the union over the orbits of the classifying spaces of the stabilizer subgroups.

\section{Definition 4.24 .}

(i) A continuous map $f: X \rightarrow Y$ of topological spaces is a weak homotopy equivalence if the induced map $f_{*}: \pi_{0} X \rightarrow \pi_{0} Y$ is an isomorphism of sets and $f_{*}: \pi_{n}(X, x) \rightarrow \pi_{n}(Y, f(x))$ is an isomorphism for all $n>0$ and all $x \in X$.

(ii) A map $F_{\bullet} \rightarrow F_{\bullet}^{\prime}$ of simplicial sets is a weak equivalence if the induced map $\left|F_{\bullet}\right| \rightarrow\left|F_{\bullet}^{\prime}\right|$ of geometric realizations is a weak homotopy equivalence.

This completes the definition of weak equivalence $\simeq$ for each of the three categories in (4.8), and they are compatible in that the image of a weak equivalence is a weak equivalence.

The simplicial set which describes $G$-connections on a fixed manifold $M$ is associated to the groupoid $\mathcal{G}(M)$ defined analogously to (4.4):

$$
\begin{gathered}
\mathcal{G}_{0}=\mathcal{G}_{0}(M)=\text { collection of } G \text {-connections }(\pi, \Theta) \text { where } \pi: P \rightarrow M, \\
\qquad \Theta \in \Omega^{1}(P ; \mathfrak{g}) \text { a connection; }
\end{gathered}
$$

$$
\begin{gathered}
\mathcal{G}_{1}=\mathcal{G}_{1}(M)=\text { collection of commutative diagrams } \\
\text { with } \varphi^{*} \Theta=\Theta^{\prime} .
\end{gathered}
$$

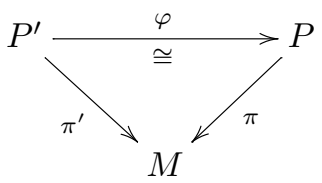

As in Example 4.11 there is an associated simplicial set $F_{\bullet}(M)$. The set of equivalence classes $\pi_{0} F_{\bullet}(M)$ of 0 -simplices is the set of equivalence classes of $G$-connections on $M$. But, as desired, $F_{\bullet}(M)$ also tracks symmetries of $G$-connections.

In summary, our response to Problem 4.1 is $\left(\operatorname{Set}_{\Delta}, \simeq\right)$ with the definition of weak equivalence $\simeq$ given in Definition 4.24 (ii). 


\section{Simplicial presheaves and Weak equivalence}

We can now address Problem 2.3 by mixing homotopy-theoretical ideas (\$4 with sheaves on manifolds ( $(3)$. We begin with the main definitions and then give many examples to illustrate.

\section{Definition 5.1.}

(i) A simplicial presheaf on manifolds (or simplicial presheaf for short) is a functor

$$
\mathcal{F}_{\bullet}: \operatorname{Man} \rightarrow \operatorname{Set}_{\Delta} .
$$

(ii) A simplicial presheaf $\mathcal{F}_{\bullet}$ is a simplicial sheaf if for each totally ordered finite set $I \in \Delta$ the presheaf of sets $\mathcal{F}_{\bullet}(I)$ is a sheaf.

(iii) For $m \in \mathbb{Z}^{\geq 0}$ the $m$-dimensional stalk of a simplicial presheaf $\mathcal{F}_{\bullet}$ is the simplicial set

$$
\underset{r \rightarrow 0}{\operatorname{colim}} \mathcal{F}_{\bullet}\left(B^{m}(r)\right)
$$

where $B^{m}(r) \subset \mathbb{A}^{m}$ is the ball of radius $r$ about the origin in $m$-dimensional affine space.

(iv) A map $\mathcal{F}_{\bullet} \rightarrow \mathcal{F}_{\bullet}^{\prime}$ of simplicial presheaves is a weak equivalence if the induced map on $m$-dimensional stalks is a weak equivalence of simplicial sets for each $m$.

We may restrict to the canonical totally ordered sets $I_{n}=\{0,1, \ldots, n\}$, as in (4.10), and so view a simplicial presheaf $\mathcal{F}_{\bullet}$ as a sequence $\mathcal{F}_{n}$ of ordinary presheaves. It is a simplicial sheaf if each $\mathcal{F}_{n}$ satisfies the sheaf condition in Definition 3.22. The $m$-dimensional stalk of $\mathcal{F}_{\bullet}$ is the simplicial set whose set of $n$-simplices is the $m$ dimensional stalk of $\mathcal{F}_{n}$. Constructions for simplicial sets carry over to simplicial sheaves. For example, a sheaf $\mathcal{G}$ of groupoids gives rise to a simplicial sheaf by applying the construction in Example 4.11 to $\mathcal{G}(M)$ for each test manifold $M$.

Example 5.4 (Discrete simplicial sheaf). Let $\mathcal{F}:$ Man $\rightarrow$ Set be a sheaf of sets on manifolds. Then as in Example 4.12 + Example 4.11 we can promote $\mathcal{F}$ to a simplicial sheaf $\widetilde{\mathcal{F}}_{\bullet}$ whose value on a test manifold $M$ is the discrete simplicial set with constant value $\mathcal{F}(M)$. We simply denote this simplicial sheaf as $\mathcal{F}$.

Example 5.5 (Representable simplicial sheaves). Recall from (3.4) that a smooth manifold $X$ gives rise to a sheaf $\mathcal{F}_{X}$. The analogue for simplicial sheaves begins with a simplicial manifold $X_{\bullet}$, which is a simplicial set

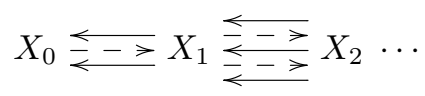

in which each $X_{n}$ is a smooth manifold and all structure maps are smooth. Let $\mathcal{F}_{X}$. be the simplicial sheaf whose value on a test manifold is the simplicial set

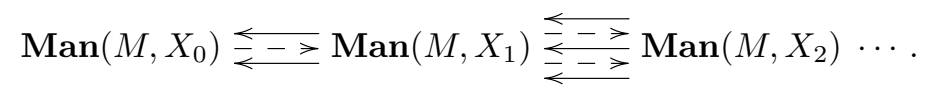

The simplicial sheaf encodes the topology of the smooth manifolds $X_{n}$; the mapping sets in (5.7) are sets of smooth maps. For example, the 0 -dimensional stalk of $\mathcal{F}_{X}$. is the simplicial set (5.6) and the $m$-dimensional stalk is the simplicial set of germs of smooth functions from an $m$-dimensional ball into the simplicial manifold (5.6). 
As a special case we consider a smooth manifold $X$ as the constant simplicial manifold

$$
X \underset{<}{\longleftarrow} X \underset{\longleftarrow-\geq}{\longleftarrow} X \cdots,
$$

where all face and degeneracy maps are identities. The induced simplicial sheaf $\mathcal{F}_{X}$ is the representable sheaf (3.4) promoted to a discrete simplicial sheaf. We emphasize that each $\mathcal{F}_{X}(M)$ is a discrete simplicial set, so is discrete as a function on totally ordered sets. But as a function of $M$ the simplicial sheaf $\mathcal{F}_{X}$ detects the smooth structure of $X$.

Let $X$ be a smooth manifold and $\mathcal{U}=\left\{U_{\alpha}\right\}_{\alpha \in A}$ an open cover. The simplicial manifold (4.17) gives rise to a representable simplicial sheaf $\left(\mathcal{F}_{\mathcal{U}}\right) \bullet$ as in Example 5.5. There is a natural map

$$
\psi:\left(\mathcal{F}_{\mathcal{U}}\right) \bullet \longrightarrow \mathcal{F}_{X}
$$

to the discrete simplicial sheaf $\mathcal{F}_{X}$ defined by inclusions $U_{\alpha_{0}} \cap \cdots \cap U_{\alpha_{n}} \subset X$.

Proposition 5.10. $\psi:\left(\mathcal{F}_{\mathcal{U}}\right) \bullet \rightarrow \mathcal{F}_{X}$ is a weak equivalence of simplicial sheaves.

Proof. Both the domain and codomain of $\psi$ are sheaves of groupoids. The inclusion map (5.9) on stalks satisfies the hypotheses of Lemma 4.3, so is an equivalence of groupoids. (See also Example 4.16.) Then Theorem 4.6] and Definition 4.24 imply that the associated simplicial sets are weakly equivalent, and so Definition [5.1(iv) implies that $\psi$ is a weak equivalence of simplicial sheaves.

We come now to our main example, which is the simplicial sheaf that classifies principal $G$-bundles with connection.

Example 5.11 $\left(B_{\nabla} G\right)$. Fix a Lie group $G$. The simplicial presheaf $B_{\nabla} G$ of $G$-connections assigns to each test manifold $M$ the simplicial set associated the groupoid (4.25) of $G$-connections on $M$. Since connections and isomorphisms of connections can be glued along open sets, the simplicial presheaf $B_{\nabla} G$ satisfies the sheaf condition. If $f: M^{\prime} \rightarrow M$ is a smooth map of manifolds, then $B_{\nabla} G(f)$ is the pullback map on $G$-connections and their isomorphisms.

Remark 5.12. There is a technical problem with this example and its close cousins below. Observe that if $M^{\prime \prime} \stackrel{f^{\prime \prime}}{\longrightarrow} M^{\prime} \stackrel{f^{\prime}}{\longrightarrow} M$ is a composition of smooth maps, and $h: M \rightarrow \mathbb{R}$ a smooth function, then the pullback is strictly associative: $\left(f^{\prime} \circ f^{\prime \prime}\right)^{*} h=\left(f^{\prime \prime}\right)^{*}\left(f^{\prime}\right)^{*} h$. However, the pullback of sets is not strictly associative. So if $E \rightarrow M$ is a fiber bundle, then while $\left(f^{\prime} \circ f^{\prime \prime}\right)^{*} E$ is canonically isomorphic to $\left(f^{\prime \prime}\right)^{*}\left(f^{\prime}\right)^{*} E$, these two fiber bundles over $M^{\prime \prime}$ are not equal. This is dealt with using Grothendieck's theory of fibered categories or alternatively higher categories.

A useful tool for verifying weak equivalences is the following.

Proposition 5.13. Let $\psi: \mathcal{F}_{\bullet} \rightarrow \mathcal{F}_{\bullet}^{\prime}$ be a map of simplicial presheaves such that $\psi(B): \mathcal{F}_{\bullet}(B) \rightarrow \mathcal{F}_{\bullet}^{\prime}(B)$ is a weak equivalence for every ball $B$ in an affine space. Then $\psi: \mathcal{F}_{\bullet} \rightarrow \mathcal{F}_{\bullet}^{\prime}$ is a weak equivalence.

We must show that $\psi$ induces a weak equivalence on stalks. As a stalk is the colimit of the values of the simplicial presheaf on balls and $\psi$ is a weak equivalence 
on balls, it suffices to show that this particular colimit7 of weak equivalences is a weak equivalence.

Now we come to the total space $E_{\nabla} G$ of the universal $G$-bundle with connection, which is the home of the universal $G$-connection.

Example 5.14 $\left(E_{\nabla} G\right)$. The simplicial sheaf $E_{\nabla} G$ attaches to any test manifold $M$ the groupoid of $G$-connections with trivialization, or rather the associated simplicial set. So $E_{\nabla} G(M)_{0}$ consists of triples $(\pi, \Theta, s)$, where $\pi: P \rightarrow M$ is a $G$-bundle, $\Theta$ is a connection, and $s: M \rightarrow P$ is a global section of $\pi$. The 1-simplices of $E_{\nabla} G(M)$ are isomorphisms $\varphi: P^{\prime} \rightarrow P$ of $G$-bundles which preserve the connection and trivialization. Higher simplices are compositions of isomorphisms, as in Example 4.11. Observe that a principal $G$-bundle with global trivialization is rigid - it has no nontrivial automorphisms. This means that $E_{\nabla} G$ is weakly equivalent to a discrete simplicial sheaf. Specifically, there are inverse weak equivalences

$$
E_{\nabla} G \rightleftarrows \Omega^{1} \otimes \mathfrak{g}
$$

where $\Omega^{1} \otimes \mathfrak{g}$ is the discrete simplicial sheaf whose value on a test manifold $M$ is $\Omega^{1}(M ; \mathfrak{g})$. On $M$ the top arrow assigns to $(\pi, \Theta, s) \in E_{\nabla} G(M)_{0}$ the 1 -form $s^{*} \Theta \in \Omega^{1}(M ; \mathfrak{g})$, and the bottom arrow assigns to $\alpha \in \Omega^{1}(M ; \mathfrak{g})$ the trivial bundle $\pi: M \times G \rightarrow M$ with identity section $s$ and connection form $\Theta=\alpha+\theta$, where $\theta$ is the Maurer-Cartan form on $G$. For each test manifold $M$ these maps determine an equivalence of groupoids (Definition 4.2) $E_{\nabla} G(M) \simeq \Omega^{1} \otimes \mathfrak{g}(M)$ : the composition beginning on the right is the identity, and in the other direction a section $s$ of a principal bundle $P \rightarrow M$ determines an isomorphism with the trivial bundle. It follows from Proposition 5.13 that (5.15) are weak equivalences of simplicial sheaves.

The notion of a smooth Lie group action on a manifold generalizes to sheaves.

Definition 5.16. Let $G$ be a Lie group and $\mathcal{F}$ a sheaf. A $G$-action on $\mathcal{F}$ is a smooth map

$$
a: \mathcal{F}_{G} \times \mathcal{F} \rightarrow \mathcal{F}
$$

which satisfies the action property: on any test manifold $M$, the sheaf map a defines an action of the group $\mathcal{F}_{G}(M)=\operatorname{Man}(M, G)$ on the set $\mathcal{F}(M)$.

As in Example 4.14 there is an associated action groupoid and so simplicial sheaf

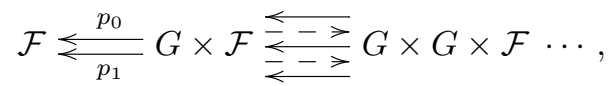

where for convenience we write ' $G$ ' in place of ' $\mathcal{F}_{G}$ '. The map $p_{0}$ is projection and $p_{1}$ is the action map (5.17).

Example $5.19\left(B_{\nabla}^{\text {triv }} G\right)$. There is a simplicial presheaf of trivializable $G$-bundles with connection, but since "trivializable" is not a local condition it is not a simplicial sheaf. It is the sub-simplicial presheaf of $B_{\nabla} G$ whose value on a test manifold $M$ consists of pairs $(\pi, \Theta)$ such that $\pi: P \rightarrow M$ admits sections (but no section is specified). We replace it by a more explicit simplicial presheaf which $i s$ a simplicial sheaf. Observe that if $\pi: P \rightarrow M$ is a principal $G$-bundle with global trivialization $s: M \rightarrow P$, then any other global trivialization is given by $s \cdot g: M \rightarrow P$ for a unique $g: M \rightarrow G$. Now the set of smooth maps $M \rightarrow G$ is the value of the sheaf $\mathcal{F}_{G}$ of

\footnotetext{
${ }^{7}$ The colimit of weak equivalences is a weak equivalence for arbitrary filtered colimits.
} 
groups on the test manifold $M$; see (3.4). Under the equivalence (5.15) the action of $g: M \rightarrow G$ on $\alpha \in \Omega^{1}(M ; \mathfrak{g})$ is given by the formula

$$
\alpha \cdot g=g^{*} \theta+\operatorname{Ad}_{g^{-1}} \alpha=g^{-1} d g+g^{-1} \alpha g .
$$

Here $\theta$ is the Maurer-Cartan form on $G$, and the last expression is valid only for matrix groups. So let $B_{\nabla}^{\text {triv }} G$ be the simplicial sheaf

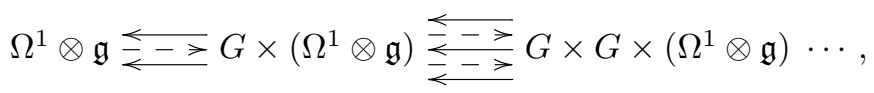

where for convenience we write ' $G$ ' in place of ' $\mathcal{F}_{G}$ '. The first two solid arrows in (5.21) are

$$
g^{*} \theta+\operatorname{Ad}_{g^{-1}} \alpha \underset{1}{\longleftarrow} g, \alpha .
$$

Note that $B_{\nabla}^{\text {triv }} G$ is a sheaf of groupoids, the action groupoid of $G$ acting on $\Omega^{1} \otimes \mathfrak{g}$.

The next result will enable us to make explicit computations with $B_{\nabla} G$ in 8 Define a map

$$
\psi: B_{\nabla}^{\text {triv }} G \longrightarrow B_{\nabla} G
$$

as the following map of sheaves of groupoids. On a test manifold $M$ it maps an element $\alpha \in \Omega^{1}(M ; \mathfrak{g})$ to the trivial bundle $\pi: M \times G \rightarrow M$ with connection form $\Theta=\alpha+\theta$. A map $g: M \rightarrow G$ induces an isomorphism of the trivial bundle with connection $\alpha+\theta$ to the trivial bundle with connection $\alpha \cdot g+\theta$, where $\alpha \cdot g$ is defined in (5.20).

Proposition 5.24. $\psi: B_{\nabla}^{\text {triv }} G \rightarrow B_{\nabla} G$ is a weak equivalence of groupoids, hence of simplicial sheaves.

Proof. We apply Proposition 5.13. On a ball $B$ any principal bundle is trivializable, so $\psi(M)$ is essentially surjective. Given $\alpha, \alpha^{\prime} \in \Omega^{1}(M ; \mathfrak{g})$ the set of $g: M \rightarrow G$ such that $\alpha^{\prime}=\alpha \cdot g$ is in bijection with automorphisms of the trivial bundle which map $\alpha+\theta$ to $\alpha \cdot g+\theta$, whence $\psi(M)$ is fully faithful.

We now state our solution to Problem 2.3. Up to the weak equivalences (5.15) and (5.23) the group $G$ (as a simplicial presheaf) acts freely on $E_{\nabla} G$ with quotient $B_{\nabla} G$, so $E_{\nabla} G \rightarrow B_{\nabla} G$ is a principal $G$-bundle. Further, there is a canonical $\mathfrak{g}$-valued 1-form on $E_{\nabla} G$, that is, a canonical map

$$
\Theta^{\text {univ }}: E_{\nabla} G \rightarrow \Omega^{1} \otimes \mathfrak{g},
$$

which is simply the top arrow in (5.15). We call $\Theta^{\text {univ }}$ the universal G-connection. That appellation is justified by the following result.

Proposition 5.26. Let $\pi: P \rightarrow X$ be a principal $G$-bundle with connection $\Theta \in$ $\Omega^{1}(P ; \mathfrak{g})$. Then there is a unique classifying map

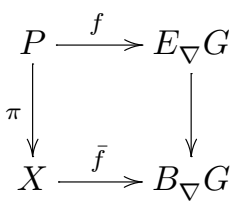

such that $f^{*} \Theta^{\text {univ }}=\Theta$. 
In (5.27) we promote $P$ and $X$ to discrete simplicial presheaves. As expected, the construction is completely tautological.

Proof. Let $\tilde{\pi}: \tilde{P} \rightarrow P$ denote the pullback of $\pi: P \rightarrow X$ by $\pi$, and $\tilde{\Theta}=\pi^{*} \Theta$ the pullback connection form on $\tilde{P}$. There is a canonical section $\tilde{s}: P \rightarrow \tilde{P}$ of $\tilde{\pi}$. The triple $(\tilde{\pi}, \tilde{\Theta}, \tilde{s})$ is a 0 -simplex in $E_{\nabla} G(P)$. On a test manifold $M$ define

$$
\begin{array}{ll}
\operatorname{Man}(M, P) \stackrel{f(M)}{\longrightarrow} & E_{\nabla} G(M) \\
(\phi: M \rightarrow P) \longmapsto & \phi^{*}(\tilde{\pi}, \tilde{\Theta}, \tilde{s}) .
\end{array}
$$

Unwinding the definitions, we verify $f^{*} \Theta^{\text {univ }}=\Theta$. The uniqueness is clear.

\section{Abstract homotopy theory}

We are now in a situation best captured by "abstract homotopy theory", or "homotopical algebra" (Q1). We have a category $\mathcal{C}$ (in our case simplicial presheaves) and a collection $\mathcal{W}$ of maps in $\mathcal{C}$ we are calling weak equivalences. These weak equivalences are not isomorphisms, but we wish to think of them as being so. We therefore focus on the invariants of weak equivalence, or more precisely functors

$$
F: \mathcal{C} \longrightarrow \mathcal{D}
$$

with the property that if $X \rightarrow Y$ is a weak equivalence in $\mathcal{C}$ then $F X \rightarrow F Y$ is an isomorphism in $\mathcal{D}$. There is a universal such functor $L: \mathcal{C} \rightarrow$ ho $\mathcal{C}$ called the localization of $\mathcal{C}$ with respect to $\mathcal{W}$. It is characterized uniquely up to unique isomorphism by the following universal property: for every category $\mathcal{D}$ and every functor $F: \mathcal{C} \rightarrow \mathcal{D}$ taking the maps in $\mathcal{W}$ to isomorphisms, there is a unique functor ho $\mathcal{C} \rightarrow D$ making the diagram

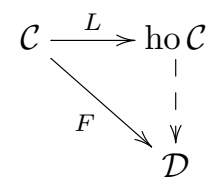

commute.

Remark 6.3. Another common notation for ho $\mathcal{C}$ is $\mathcal{W}^{-1} \mathcal{C}$. Our choice of 'ho $\mathcal{C}$ ', and the nomenclature 'homotopy category' we adopt for it, emerges in Example 6.5 below.

The localization ho $\mathcal{C}$ is constructed by freely adding to $\mathcal{C}$, for each $f \in \mathcal{W}$, a new morphism $f^{-1}$, and imposing the relations $f^{-1} \circ f=\mathrm{id}$ and $f \circ f^{-1}=\mathrm{id}$. The issue then becomes to somehow describe the new collection of maps

$$
\operatorname{ho} \mathcal{C}(X, Y)
$$

for each $X, Y \in \mathcal{C}$. In general, there is not a guarantee that this is even a set.

Let us look at some examples.

Example 6.5. Suppose first that $\mathcal{C}$ is the category of $\mathrm{CW}$ complexes, and that $\mathcal{W}$ is the collection of maps which are weak homotopy equivalences, i.e., maps $f: X \rightarrow Y$ with the property that for each point $x \in X$ the map of homotopy groups

$$
\pi_{n}(X, x) \longrightarrow \pi_{n}(Y, f(x))
$$

is a bijection for all $n \geq 0$ (Definition 4.24). Let $\pi \mathcal{C}$ be the homotopy category of $\mathcal{C}$ : the set of maps $\pi \mathcal{C}(X, Y)$ is the quotient of $\mathcal{C}(X, Y)$ by the equivalence relation 
which identifies homotopic maps. By the Whitehead Theorem, weak equivalences between $\mathrm{CW}$ complexes are homotopy equivalences, so the maps in $\mathcal{W}$ are sent to isomorphisms in $\pi \mathcal{C}$. The universal property of ho $\mathcal{C}$ then provides a unique functor

$$
\text { ho } \mathcal{C} \longrightarrow \pi \mathcal{C}
$$

factoring the quotient $\mathcal{C} \rightarrow \pi \mathcal{C}$. On the other hand, the inclusions

$$
\begin{aligned}
& X \times\{0\} \longrightarrow X \times[0,1], \\
& X \times\{1\} \longrightarrow X \times[0,1]
\end{aligned}
$$

are in $\mathcal{W}$, so homotopic maps in $\mathcal{C}$ go to the same map in ho $\mathcal{C}$. This shows that the functor $\mathcal{C} \rightarrow$ ho $\mathcal{C}$ factors uniquely through $\pi \mathcal{C}$. It follows that (6.7) is in fact an isomorphism of categories. Thus the maps in ho $\mathcal{C}$ in this case may be calculated as homotopy classes of maps.

The terminology of homotopical algebra Q1 is borrowed from this example. In the language of abstract homotopy theory, the class of maps $\mathcal{W}$ is called the class of "weak equivalences" and the category ho $\mathcal{C}$ is called the "homotopy category" of $\mathcal{C}$. We will now use this terminology. Often there is a notion of "homotopy" floating around in $\mathcal{C}$, and we will use the symbol ' $\pi(X, Y)$ ' to denote the quotient of $\mathcal{C}(X, Y)$ by the equivalence relation generated by homotopy. Generally one hopes to describe ho $\mathcal{C}(X, Y)$ in terms of $\pi(X, Y)$.

Example 6.9. Let us look at another example. Let $R$ be a ring and Chain $_{R}$ the category of nonnegatively graded chain complexes

$$
\longrightarrow C_{n} \stackrel{d}{\longrightarrow} C_{n-1} \longrightarrow \cdots \longrightarrow C_{0} .
$$

We take the class of weak equivalences $\mathcal{W}$ to be the class of maps inducing an isomorphism of homology groups. The notion of homotopy we have floating around is that of chain homotopy, and we define $\pi(X, Y)$ to be the set of chain homotopy classes of maps from $X$ to $Y$. Let $I$ denote the chain complex of free abelian groups whose only nonzero terms are in degrees 0 and 1 , and in those degrees is given by

$$
\begin{aligned}
\mathbb{Z}\{h\} & \longrightarrow \mathbb{Z}\left\{e_{0}, e_{1}\right\} \\
h & \longmapsto e_{1}-e_{0} .
\end{aligned}
$$

Then a chain homotopy is a map $X \otimes I \rightarrow Y$. Now the two maps

$$
X \otimes \mathbb{Z}\left\{e_{i}\right\} \longrightarrow X \otimes I
$$

are weak equivalences, so chain homotopic maps are identified in ho Chain . This provides a natural map $\pi(X, Y) \rightarrow$ ho $\operatorname{Chain}_{R}(X, Y)$. By basic homological algebra, if $X$ is a chain complex of projective $R$-modules and $Y \rightarrow Z$ is a weak equivalence then $\pi(X, Y) \rightarrow \pi(X, Z)$ is an isomorphism. Thus in this case the functor

$$
\pi(X,-): \text { Chain }_{R} \longrightarrow \mathbf{A b}
$$

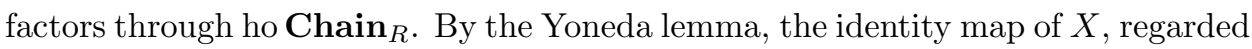
as an element of $\pi(X, X)$, gives a natural (in $Y$ ) map ho $\operatorname{Chain}_{R}(X, Y) \rightarrow \pi(X, Y)$. It is straightforward to check that the composites are both the identity. So when $X$ is a complex of projectives, then ho $\operatorname{Chain}_{R}(X, Y)$ is given by $\pi(X, Y)$. For a 
general $X$ one can always find a weak equivalence $\tilde{X} \rightarrow X$ from a complex of projectives to $X$ (a projective resolution). One then has the sequence of isomorphisms

$$
\operatorname{ho}_{C^{\prime}} \operatorname{chain}_{R}(X, Y) \approx \text { ho } \operatorname{Chain}_{R}(\tilde{X}, Y) \approx \pi(\tilde{X}, Y) .
$$

Turning to the case of interest to us, let sPre be the category of simplicial presheaves (or, equivalently, presheaves of simplicial sets) on the category Man of smooth manifolds. Recall Definition 5.1(iv): a map $\mathcal{F}_{\bullet} \rightarrow \mathcal{F}_{\bullet}^{\prime}$ of simplicial presheaves is a weak equivalence if the induced map of stalks is a weak equivalence of simplicial sets.

We begin our analysis of ho $\operatorname{sPre}\left(\mathcal{F}_{\bullet}, \mathcal{F}_{\bullet}^{\prime}\right)$ with the special case in which $\mathcal{F}_{\bullet}^{\prime}=\mathcal{F}^{\prime}$ is a sheaf, regarded as a constant simplicial presheaf. Write $\underline{\pi}_{0} \mathcal{F}$. for the sheaf associated to the presheaf

$$
M \longmapsto \pi_{0}\left(\mathcal{F}_{\bullet}(M)\right),
$$

so that $\underline{\pi}_{0} \mathcal{F}_{\bullet}$ is the sheaf associated to the presheaf obtained as the coequalizer of

$$
\mathcal{F}_{1} \underset{d_{1}}{\stackrel{d_{0}}{\longrightarrow}} \underset{\mathcal{F}_{0}}{ } \text {. }
$$

(See Definition 4.18) Two facts about $\underline{\pi}_{0}$ follow readily from the definition. One is that a weak equivalence $\widetilde{\mathcal{F}}_{\bullet} \rightarrow \mathcal{F}_{\bullet}$ induces an isomorphism $\underline{\pi}_{0} \widetilde{\mathcal{F}}_{\bullet} \rightarrow \underline{\pi}_{0} \mathcal{F}_{\bullet}$, so that $\underline{\pi}_{0}$ defines a functor on hosPre. The other is that the set of maps of simplicial presheaves

$$
\mathcal{F}_{\bullet} \longrightarrow \mathcal{F}^{\prime}
$$

is naturally isomorphic to the set of sheaf maps

$$
\underline{\pi}_{0} \mathcal{F}_{\bullet} \longrightarrow \mathcal{F}^{\prime}
$$

From this it follows that

$$
\mathcal{F}_{\bullet} \longmapsto \operatorname{sPre}\left(\mathcal{F}_{\bullet}, \mathcal{F}^{\prime}\right)
$$

factors through hosPre, and as in our analysis of chain complexes, that the map

$$
\operatorname{sPre}\left(\mathcal{F}_{\bullet}, \mathcal{F}^{\prime}\right) \stackrel{\cong}{h} \operatorname{sire}\left(\mathcal{F}_{\bullet}, \mathcal{F}^{\prime}\right)
$$

is a bijection.

Now suppose that $\mathcal{F}_{\bullet}^{\prime}=\mathcal{P}$ is a presheaf, regarded as a constant simplicial presheaf, and let $\mathbf{a} \mathcal{P}$ be the associated sheaf. Then the canonical map $\mathcal{P} \rightarrow \mathbf{a} P$ is a weak equivalence (it is an isomorphism on stalks, as stated in Remark 3.28). It follows that one may compute ho $\mathcal{C}\left(\mathcal{F}_{\bullet}, \mathcal{P}\right)$ via the isomorphisms

$$
\operatorname{hos} \operatorname{Pre}\left(\mathcal{F}_{\bullet}, \mathcal{P}\right) \approx \operatorname{hos} \operatorname{Pre}\left(\mathcal{F}_{\bullet}, \mathbf{a} \mathcal{P}\right) \approx \operatorname{sPre}\left(\mathcal{F}_{\bullet}, \mathbf{a} P\right) .
$$

Summarizing, we have

Proposition 6.22. Let $\mathcal{P}$ be a presheaf, regarded as a constant simplicial presheaf, and $\mathbf{a} \mathcal{P}$ be the associated sheaf. Then for any $\mathcal{F}_{\bullet} \in \mathbf{s P r e}$, the maps

$$
\operatorname{hos} \operatorname{Pre}\left(\mathcal{F}_{\bullet}, \mathcal{P}\right) \approx \operatorname{hos} \operatorname{Pre}\left(\mathcal{F}_{\bullet}, \mathbf{a} \mathcal{P}\right) \approx \operatorname{sPre}\left(\mathcal{F}_{\bullet}, \mathbf{a} \mathcal{P}\right)
$$

are isomorphisms. In particular, if $\mathcal{P}=\mathcal{F}^{\prime}$ is a sheaf, regarded as a constant simplicial presheaf, then the map

$$
\operatorname{sPre}\left(\mathcal{F}_{\bullet}, \mathcal{F}^{\prime}\right) \longrightarrow \operatorname{hosPre}\left(\mathcal{F}_{\bullet}, \mathcal{F}^{\prime}\right)
$$

is an isomorphism. 
Note that the domain $\operatorname{sPre}\left(\mathcal{F}_{\bullet}, \mathcal{F}^{\prime}\right)$ of $(\underline{6.24})$ is the equalizer of

$$
\operatorname{Pre}\left(\mathcal{F}_{0}, \mathcal{F}^{\prime}\right) \longrightarrow \operatorname{Pre}\left(\mathcal{F}_{1}, \mathcal{F}^{\prime}\right) \text {. }
$$

Remark 6.26. If $\mathcal{P}$ is a constant simplicial presheaf, then $\underline{\pi}_{0} \mathcal{P}$ is just the associated sheaf a $\mathcal{P}$. When $\mathcal{P}$ and $\mathcal{P}^{\prime}$ are presheaves, regarded as constant simplicial presheaves, Proposition 6.22 therefore provides an isomorphism

$$
\operatorname{sPre}\left(\mathbf{a} \mathcal{P}, \mathbf{a} \mathcal{P}^{\prime}\right) \approx \operatorname{hos} \operatorname{Pre}\left(\mathcal{P}, \mathcal{P}^{\prime}\right) .
$$

Put differently, the homotopy theory of simplicial presheaves knows about sheaves, so even if we were only interested in sheaves and presheaves, working in the homotopy theory of simplicial presheaves incorporates the fundamental relationship between them. This highlights one role played by abstract homotopy theory. It can be used to locate objects constrained by algebraic conditions, like the sheaf condition, within a broader context more suitable for doing homotopy theory.

Proposition 6.22 computes the maps in the homotopy category of presheaves when the codomain is a constant simplicial presheaf. This is what is used in the remainder of this paper. A general formula is the content of the Verdier Hypercovering Theorem. We do not attempt a complete exposition, and the reader may skip the remainder of this section without penalty.

We begin with a motivating example.

Example 6.28. Let $X$ be a smooth manifold and $\mathcal{F}_{X}$ the associated discrete simplicial sheaf. A map $\mathcal{F}_{X} \rightarrow B_{\nabla}^{\text {triv }} G$ is a connection on the trivial $G$-bundle over $X$. Let $\mathcal{U}$ be an open cover of $X$ and $\left(\mathcal{F}_{\mathcal{U}}\right)$. the associated representable simplicial sheaf. Then a map $\left(\mathcal{F}_{\mathcal{U}}\right) \bullet \rightarrow B_{\nabla}^{\text {triv }} G$ is a $G$-bundle with connection on $X$ together with a trivialization on each open set of the cover. Homotopic maps give isomorphic $G$-bundles with connection. Since any principal $G$-bundle admits local trivializations, the colimit

$$
\operatorname{colim} \pi\left(\left(\mathcal{F}_{\mathcal{U}}\right)_{\bullet}, B_{\nabla}^{\text {triv }} G\right) \approx \operatorname{hos} \operatorname{Pre}\left(\mathcal{F}_{X}, B_{\nabla} G\right)
$$

over all open covers $\mathcal{U}$ is in bijection with the set of isomorphism classes of $G$ connections on $X$. Then applying Proposition 5.24 we deduce an isomorphism

$$
\operatorname{colim}_{\mathcal{U}} \pi\left(\left(\mathcal{F}_{\mathcal{U}}\right)_{\bullet}, B_{\nabla}^{\text {triv }} G\right) \approx \operatorname{hos} \operatorname{Pre}\left(\mathcal{F}_{X}, B_{\nabla}^{\text {triv }} G\right) .
$$

This is a special case of the Verdier theorem in which covers, rather than hypercovers, suffice to compute maps in the homotopy category.

We now state the general result. Let Hypercov $_{\mathcal{F}_{\mathfrak{*}}}$ be the category of hypercovers of a simplicial presheaf $\mathcal{F}_{\bullet}$ and (simplicial) homotopy classes of maps. Given a simplicial presheaf $\mathcal{F}_{\bullet}^{\prime}$, form

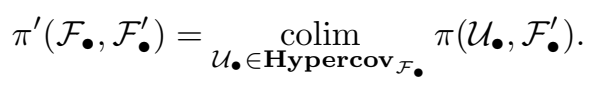

Since each hypercovering $\mathcal{U}_{\bullet} \rightarrow \mathcal{F}_{\bullet}$ is a weak equivalence, there is a natural map

$$
\pi^{\prime}\left(\mathcal{F}_{\bullet}, \mathcal{F}_{\bullet}^{\prime}\right) \longrightarrow \operatorname{hos} \operatorname{Pre}\left(\mathcal{F}_{\bullet}, \mathcal{F}_{\bullet}^{\prime}\right) \text {. }
$$

Theorem 6.33 (Verdier hypercovering theorem). If $\mathcal{F}_{\bullet}^{\prime}$ is stalkwise a Kan complex, then the map

$$
\pi^{\prime}\left(\mathcal{F}_{\bullet}, \mathcal{F}_{\bullet}^{\prime}\right) \longrightarrow \operatorname{hos} \operatorname{Pre}\left(\mathcal{F}_{\bullet}, \mathcal{F}_{\bullet}^{\prime}\right)
$$

is a bijection. 
The Verdier hypercovering theorem is an elaboration of Verdier's Théorème 7.4.1 in SGA. It was originally formulated in the above manner in the thesis of Ken Brown $[\mathrm{Br}$ (see specifically Example 2, Theorem 1, and Theorem 2). It was further extended and refined by Jardine (see [J] and the references therein).

Remark 6.35. The Verdier hypercovering theorem contains, as a special case, a formula for describing a $\mathcal{P}$ in terms of $\mathcal{P}$.

Abstract homotopy theory was introduced by Quillen in Q1 and Q2 under the name of "Model Categories". His original applications were to finding an algebraic model for rational homotopy theory and for defining the "cotangent complex" of a map of commutative rings, which plays the role of the cotangent bundle when the map is not smooth. In the original applications the emphasis was on the systematic comparison of resolutions, and alongside the weak equivalences Quillen specified two further collections of maps, the cofibrations and fibrations, and some properties relating the three classes. In terms of these he defined a notion of homotopy, and produced the general formula

$$
\operatorname{ho} \mathcal{C}(X, Y) \approx \pi\left(X^{\prime}, Y^{\prime}\right) .
$$

Here $X^{\prime}$ is an object equipped with a weak equivalence $X^{\prime} \rightarrow X$ and having the property that the unique map it receives from the initial object $\emptyset \rightarrow X$ is a cofibration, $Y^{\prime}$ is an object equipped with a weak equivalence $Y \rightarrow Y^{\prime}$ and having the property that its unique map $Y^{\prime} \rightarrow *$ to the terminal object is a fibration, and $\pi\left(X^{\prime}, Y^{\prime}\right)$ is the quotient of $\mathcal{C}\left(X^{\prime}, Y^{\prime}\right)$ by the relation of homotopy. One thinks of $X^{\prime}$ as analogous to a projective resolution of $X$ and $Y^{\prime}$ as analogous to an injective resolution of $Y$. The use of homotopical algebra to implement algebraic structures originates in the work of Bousfield $\mathrm{Bo1}, \mathrm{Bo2}$ and plays an important role in the study of the moduli spaces of interest in homotopy theory. The special role played by the weak equivalences was apparent early on, but it was Kan et al. DHKS who undertook to do homotopical algebra solely with the weak equivalences. A good introduction to Model Categories can be found in [DS].

\section{The de Rham complex of $B_{\nabla} G$}

At last the abstractions and tautologies give way to theorems.

Definition 7.1. Let $\mathcal{F}_{\bullet}$ be a simplicial presheaf. The de Rham complex of $\mathcal{F}_{\bullet}$ is

$$
\begin{aligned}
& \operatorname{hos} \operatorname{Pre}\left(\mathcal{F}_{\bullet}, \Omega^{0} \stackrel{d}{\longrightarrow} \Omega^{1} \stackrel{d}{\longrightarrow} \cdots\right) \\
& \quad \cong \operatorname{hos} \operatorname{Pre}\left(\mathcal{F}_{\bullet}, \Omega^{0}\right) \stackrel{d}{\longrightarrow} \operatorname{hos} \operatorname{Pre}\left(\mathcal{F}_{\bullet}, \Omega^{1}\right) \stackrel{d}{\longrightarrow} \cdots .
\end{aligned}
$$

By Proposition 6.22 each term in the complex (7.2) can be computed as an equalizer 6.25):

$$
\operatorname{hos} \operatorname{Pre}\left(\mathcal{F}_{\bullet}, \Omega^{n}\right)=\operatorname{ker}\left\{\Omega^{n}\left(\mathcal{F}_{0}\right) \underset{\rho_{1}}{\stackrel{\rho_{0}}{\longrightarrow}} \Omega^{n}\left(\mathcal{F}_{1}\right)\right\}
$$

where $\rho_{0}, \rho_{1}$ are induced from the structure maps $\mathcal{F}_{0} \underset{p_{1}}{\longleftarrow} \mathcal{F}_{1}$.

Remark 7.4. It follows from Definition 7.1 that weakly equivalent simplicial presheaves have isomorphic de Rham complexes. Therefore, the de Rham complex is a homotopy invariant. Below we compute the de Rham complex of $B_{\nabla} G$ using a convenient weakly equivalent simplicial presheaf, based on Proposition 5.24. 
Proposition 7.5. Suppose a Lie group $G$ acts on a sheaf $\mathcal{F}$. Then the de Rham complex of the associated simplicial sheaf

$$
\mathcal{F} \underset{p_{1}}{\longleftarrow} G \times \mathcal{F} \underset{p_{0}}{\stackrel{-}{\longleftarrow}} G \times G \times \mathcal{F} \ldots
$$

is the equalizer

$$
\operatorname{ker}\left\{\Omega^{\bullet}(\mathcal{F}) \underset{\rho_{1}}{\stackrel{\rho_{0}}{\longrightarrow}} \rightarrow \Omega^{\bullet}(G \times \mathcal{F})\right\} .
$$

This is immediate from (7.3).

The equalizer of $\rho_{0}$ and $\rho_{1}$ has an interpretation as the basic subcomplex $\Omega^{\bullet}(\mathcal{F})_{\text {bas }} \subset \Omega^{\bullet}(\mathcal{F})$, which we now define. We begin by reviewing some standard constructions in de Rham theory. Suppose $G$ is a Lie group which acts smoothly on a manifold $X$. Then the infinitesimal action associates to each $\xi \in \mathfrak{g}=\operatorname{Lie}(G)$ a vector field $\hat{\xi}$ on $X$, and so a contraction operator

$$
\iota_{\hat{\xi}}: \Omega^{\bullet}(X) \rightarrow \Omega^{\bullet-1}(X)
$$

of degree -1 and a Lie derivative

$$
\mathcal{L}_{\hat{\xi}}: \Omega^{\bullet}(X) \rightarrow \Omega^{\bullet}(X)
$$

of degree 0 . They are related by Cartan's formula $\mathcal{L}_{\hat{\xi}}=d \iota_{\hat{\xi}}+\iota_{\hat{\xi}} d$. Replace $X$ by a sheaf $\mathcal{F}$. Fix $\omega \in \Omega^{\bullet}(\mathcal{F})$. Then for any test manifold $M$ and $\phi \in \mathcal{F}(M)$, we obtain $\omega(M, \phi) \in \Omega^{\bullet}(M)$. The action $a$ determines a map

$$
G \times M \stackrel{\operatorname{id} \times \phi}{\longrightarrow} G \times \mathcal{F} \stackrel{a}{\longrightarrow} \mathcal{F} \stackrel{\omega}{\longrightarrow} \Omega^{\bullet},
$$

which is the differential form $\eta=a^{*} \omega(G \times M$, id $\times \phi) \in \Omega^{\bullet}(G \times M)$. (As usual we write ' $G$ ' for ' $\mathcal{F}_{G}$ '.) For $\xi \in \mathfrak{g}$ define $\iota_{\xi} \omega \subset \Omega^{\bullet-1}(\mathcal{F})$ by

$$
\iota_{\xi} \omega(M, \phi)=\left.\iota_{\bar{\xi}} \eta\right|_{\{e\} \times M},
$$

where $\bar{\xi}$ is the vector field on $G \times M$ defined by $\xi$ (it points along the $G$-factor) and $e \in G$ is the identity element. The Lie derivative $\mathcal{L}_{\xi} \omega$ is defined by a formula similar to (7.11):

$$
\mathcal{L}_{\xi} \omega(M, \phi)=\left.\mathcal{L}_{\bar{\xi}} \eta\right|_{\{e\} \times M} .
$$

The Lie derivative and contraction satisfy Cartan's formula.

Definition 7.13. Suppose a Lie group $G$ acts on a sheaf $\mathcal{F}$. Then the differential form $\omega \in \Omega^{\bullet}(\mathcal{F})$ is basic if (i) $\left.a^{*} \omega\right|_{\{g\} \times \mathcal{F}}=\omega$ for all $g \in G$, and (ii) $\iota_{\xi} \omega=0$ for all $\xi \in \mathfrak{g}$.

Note that (i) is the condition that $\omega$ be $G$-invariant. The differential of a basic form is basic, so basic forms comprise a subcomplex $\Omega^{\bullet}(\mathcal{F})_{\text {bas }} \subset \Omega^{\bullet}(\mathcal{F})$. Condition (ii) can be rephrased in terms of a global condition on $G \times \mathcal{F}$.

Proposition 7.14. The equalizer (7.7) is the basic subcomplex $\Omega^{\bullet}(\mathcal{F})_{\text {bas }}$.

Proof. Recall that $p_{0}$ is projection and $p_{1}$ is the action map $a$; then $\rho_{0}, \rho_{1}$ are the respective pullbacks. We claim that for any (test) manifold $M$ a form $\omega \in \Omega^{\bullet}(M)$ is basic if and only if $p_{0}^{*} \omega=p_{1}^{*} \omega \in \Omega^{\bullet}(G \times M)$, where $p_{0}: G \times M \rightarrow M$ is projection and $p_{1}: G \times M \rightarrow M$ is the action. We make two remarks to aid the reader in the verification. First, an element $\xi \in \mathfrak{g}$ induces a vector field $\hat{\xi}$ on $M$ and a vector 
field $\bar{\xi}$ on $G \times M$, and these vector fields are "related" by the action map $p_{1}$ : in other words, $\left(p_{1}\right)_{*}(\bar{\xi})=\hat{\xi}$. It follows that for any $\omega \in \Omega^{\bullet}(M)$, we have $\iota_{\bar{\xi}} p_{1}^{*} \omega=p_{1}^{*} \iota_{\hat{\xi}} \omega$. Second, a form $\eta \in \Omega^{\bullet}(G \times M)$ is pulled back from $M$ if and only if $\eta$ is $G$-invariant and $\iota \bar{\xi} \eta=0$ for all $\xi \in \mathfrak{g}$.

We introduce the following standard differential graded algebra.

Definition 7.15. Let $V$ be a real vector space. Then the Koszul complex $\operatorname{Kos}^{\bullet} V$ built on $V$ is the differential graded algebra

$$
\operatorname{Kos}^{\bullet} V=\bigwedge^{\bullet} V \otimes \operatorname{Sym}^{\bullet} V
$$

with differential

$$
d_{K}(v)=\tilde{v}, \quad d_{K}(\tilde{v})=0, \quad v \in V=\bigwedge^{1} V, \quad \tilde{v} \in V=\operatorname{Sym}^{1} V .
$$

Here ' $\tilde{v}$ ' denotes $v \in V$ regarded as an element of $\operatorname{Sym}^{1} V$. Note that $\operatorname{Kos}^{\bullet} V$ is generated by $V=\wedge^{1} V$ as a differential graded algebra. We grade the generators by $\operatorname{deg} \bigwedge^{1} V=1$; it follows that $\operatorname{deg} \operatorname{Sym}^{1} V=2$. It is a standard result that $\operatorname{Kos}^{\bullet} V$ has trivial cohomology:

$$
H^{\bullet}\left(\operatorname{Kos} V, d_{K}\right)=\mathbb{R} .
$$

This follows for finite-dimensional $V$ (which is all we need) from the isomorphism $\operatorname{Kos}^{\bullet}\left(V_{1} \oplus V_{2}\right) \cong \operatorname{Kos}^{\bullet} V_{1} \otimes \operatorname{Kos}^{\bullet} V_{2}$ and the case when $V$ is one dimensional.

Our first result is the following.

Theorem 7.19. The de Rham complex of $E_{\nabla} G$ is $\left(\operatorname{Kos}^{\bullet} \mathfrak{g}^{*}, d_{K}\right)$.

It follows from (17.18) that the de Rham cohomology of $E_{\nabla} G$ is that of a contractible manifold. $\operatorname{Kos}^{\bullet} \mathfrak{g}^{*}$ is called the Weil algebra of the Lie algebra $\mathfrak{g}$. Henri Cartan C1, C2 used the Weil algebra as a model for the cohomology of any realization of $E G$ as a space. The novelty here is that the Weil algebra is precisely the de Rham complex of the generalized manifold $E_{\nabla} G$.

Next we state the solution to Problem 2.4

Theorem 7.20. The de Rham complex of $B_{\nabla} G$ is $\left(\left(\operatorname{Sym}_{\bullet} \mathfrak{g}^{*}\right)^{G}, d=0\right)$.

Here $\operatorname{Sym}^{\bullet}\left(\mathfrak{g}^{*}\right)^{G}$ is the space of Ad-invariant polynomials on $\mathfrak{g}$, graded by twice the degree, and the de Rham differential vanishes on $B_{\nabla} G$. The classical Chern-Weil homomorphism [CS] is an injection

$$
\left(\left(\operatorname{Sym}^{\bullet} \mathfrak{g}^{*}\right)^{G}, d=0\right) \longrightarrow\left(\Omega^{\bullet}\left(B_{\nabla} G\right), d\right) .
$$

Namely, given an invariant polynomial of degree $k$ on $\mathfrak{g}$ we apply it to the curvature of a $G$-connection to obtain a closed $2 k$-form, and this construction is local and natural. Theorem 7.20 asserts that Chern-Weil forms are the only natural differential forms attached to a $G$-connection.

The proofs of Theorem 7.19 and Theorem 7.20 are given in $\$ 8$.

Let $X$ be a smooth manifold with a left action of the Lie group $G$. The Borel quotient, or homotopy quotient, of $X$ is usually defined as $X_{G}=E G \times_{G} X$, where $E G$ is a contractible space on which $G$ acts freely, and $X_{G}$ is the quotient of $E G \times X$ by the diagonal $G$-action. We mimic this construction in the world of simplicial sheaves.

Definition 7.22. The simplicial Borel quotient of $X$ by $G$ is the simplicial sheaf representing the action of $G$ on $E_{\nabla} G \times X$. 
Using the equivalence (5.15) of $E_{\nabla} G$ with $\Omega^{1} \otimes \mathfrak{g}$, the simplicial Borel quotient is equivalent to the simplicial sheaf $\left(X_{G}\right)_{\nabla}$ indicated in the diagram (cf. (5.21) $)$

$$
\left(\Omega^{1} \otimes \mathfrak{g}\right) \times X \gtreqless-\gtrless G \times\left(\Omega^{1} \otimes \mathfrak{g}\right) \times X \underset{\leftarrow-\gtreqless}{\longleftarrow} G \times G \times\left(\Omega^{1} \otimes \mathfrak{g}\right) \times X \cdots
$$

The first two solid arrows in (7.23) are

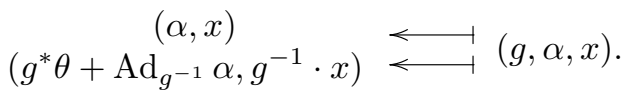

Note that $\left(X_{G}\right)_{\nabla}$ is a sheaf of groupoids.

To state the next theorem we need a preliminary lemma about the Weil algebra. The coadjoint action $\mathrm{Ad}^{*}$ of $G$ on $\mathfrak{g}^{*}$ induces an action of $G$ on the Weil algebra $\operatorname{Kos}^{\bullet} \mathfrak{g}^{*}$.

Lemma 7.25. For each $\xi \in \mathfrak{g}$, the action (5.21) of $G$ on $\Omega^{1} \otimes \mathfrak{g}$ induces a contraction $\iota_{\xi}$ on $\Omega^{\bullet}\left(\Omega^{1} \otimes \mathfrak{g}\right) \cong \operatorname{Kos}^{\bullet} \mathfrak{g}^{*}$ which satisfies

$$
\begin{aligned}
\iota_{\xi} \lambda & =\langle\xi, \lambda\rangle, & & \lambda \in \mathfrak{g}^{*}=\bigwedge^{1} \mathfrak{g}^{*}, \\
\iota_{\xi} \tilde{\lambda} & =-\operatorname{Ad}_{\xi}^{*} \lambda, & & \tilde{\lambda} \in \mathfrak{g}^{*}=\operatorname{Sym}^{1} \mathfrak{g}^{*},
\end{aligned}
$$

on generators.

We defer the proof to 98

Remark 7.27. The contraction (7.26) is usually a definition ( $\mathrm{C1}$, [C2, MQ, §5], GS, §3.2]). In our approach with sheaves it is a computation from the general definition (7.11).

\section{Theorem 7.28.}

(i) For any smooth manifold $X$ the de Rham complex of $X \times\left(\Omega^{1} \otimes \mathfrak{g}\right)$ is $\Omega\left(X ; \operatorname{Kos}^{*}\right)^{\bullet}$ with differential the sum of the de Rham differential $d_{X}$ on $X$ and the Koszul differential $d_{K}$ in (7.17).

(ii) The de Rham complex of the simplicial Borel quotient $\left(X_{G}\right)_{\nabla}$ in (7.23) is the basic subcomplex of $\Omega\left(X ; \operatorname{Kos}^{*}\right)^{\bullet}$ with differential $d_{X}+d_{K}$.

Part (i) would follow immediately from Theorem 7.19 if we prove that the de Rham complex of a Cartesian product of sheaves is the (completed) tensor product of the de Rham complexes of the factors. We give a direct proof in $\$ 8$. Part (ii) is an immediate corollary of part (i) and Proposition 7.14. The complex in Theorem[7.28(ii) is the Weil model for equivariant cohomology; see [C1, [C2, [AB, §4], MQ, §5], [GS, §4]. We realize it precisely as the de Rham complex of a generalized manifold, the simplicial sheaf $\left(X_{G}\right)_{\nabla}$.

\section{Proofs}

We prove the following slight generalization of Theorem 7.19, (Recall the weak equivalence (5.15).)

Theorem 8.1. Let $V$ be a finite-dimensional real vector space. Then the de Rham complex of $\Omega^{1} \otimes V$ is $\left(\operatorname{Kos}^{\bullet} V^{*}, d_{K}\right)$.

More precisely, we define a homomorphism of differential graded algebras

$$
\eta: \operatorname{Kos}^{\bullet} V^{*} \longrightarrow \Omega^{\bullet}\left(\Omega^{1} \otimes V\right)
$$


as follows. To $\ell \in V^{*}=\bigwedge^{1} V^{*}$ we assign the 1 -form $\eta(\ell): \Omega^{1} \otimes V \rightarrow \Omega^{1}$ characterized by

$$
\eta(\ell): \sum_{i} \alpha_{i} \otimes v_{i} \longmapsto \sum_{i}\left\langle v_{i}, \ell\right\rangle \alpha_{i}, \quad \alpha_{i} \in \Omega^{1}, v_{i} \in V .
$$

This determines the entire homomorphism $\eta$. For example, it follows that

$$
\begin{gathered}
\eta\left(\ell_{1} \wedge \ell_{2}\right)=\eta\left(\ell_{1}\right) \wedge \eta\left(\ell_{2}\right): \sum_{i} \alpha_{i} \otimes v_{i} \longmapsto \sum_{i, j}\left\langle v_{i}, \ell_{1}\right\rangle\left\langle v_{j}, \ell_{2}\right\rangle \alpha_{i} \wedge \alpha_{j} \\
\eta(\tilde{\ell})=d \eta(\ell): \sum_{i} \alpha_{i} \otimes v_{i} \longmapsto \sum_{i}\left\langle v_{i}, \ell\right\rangle d \alpha_{i} .
\end{gathered}
$$

Recall from (7.17) the notation $\tilde{\ell} \in V^{*}=\operatorname{Sym}^{1} V^{*}$. Notice that the map $\Omega^{1} \otimes V \rightarrow$ $\Omega^{\bullet}$ attached to an element of $\operatorname{Kos}^{\bullet} V^{*}$ is polynomial but not usually linear. Also, these formulas are most easily understood by pulling back to a test manifold $M$ via a map $M \rightarrow \Omega^{1} \otimes V$, in which case $\alpha_{i} \in \Omega^{1}(M)$. Theorem 8.1 asserts that $\eta$ is an isomorphism.

We present the proof of Theorem 8.1 as a series of lemmas. Recall that if $E \rightarrow M$ is a vector bundle, then for each integer $q \geq 0$ there is an associated bundle of $q$-jets $J^{q} E \rightarrow M$ defined as follows. Let $I_{p} \subset C^{\infty}(M)$ denote the ideal of functions that vanish at $p$. Then the fiber of $J^{q} E$ at $p$ is $\Omega^{0}(M ; E) / I_{p}^{q+1} \Omega^{0}(M ; E)$, the quotient of the space of sections of $E$ by the space of sections that vanish at $p$ to order $\geq q+1$. An element $\omega \in \Omega^{q}\left(\Omega^{1} \otimes V\right)$ is, for each test manifold $M$, a map of sets $\omega_{M}: \Omega^{1}(M ; V) \rightarrow \Omega^{q}(M)$, functorial in $M$.

Lemma 8.6. Fix $\omega \in \Omega^{q}\left(\Omega^{1} \otimes V\right)$. For $\alpha \in \Theta^{1}(M ; V)$ the value of $\omega_{M}(\alpha)$ at a point $p \in M$ depends only on the q-jet of $\alpha$ at $p$.

Proof. Assume first $q=0$ and $\alpha \in \Omega^{1}(M ; V)$. Let $i_{p}:\{p\} \hookrightarrow M$ denote the inclusion. Then the presheaf property (naturality) implies $\left.\omega_{M}(\alpha)\right|_{p}=i_{p}^{*} \omega_{M}(\alpha)=$ $\omega_{\{p\}}\left(i_{p}^{*} \alpha\right)=\omega_{\mathrm{pt}}(0)=\left.\omega_{M}(0)\right|_{p}$. Thus $\omega_{M}(\alpha)$ is a constant function on $M$ independent of $\alpha$ (so does not even depend on the 0 -jet of $\alpha$ ).

Now let $q>0$. Naturality implies that if $i_{U}: U \hookrightarrow M$ is the inclusion of a neighborhood of $p$ and $\alpha_{1}, \alpha_{2} \in \Omega^{1}(M ; V)$ satisfy $i_{U}^{*} \alpha_{1}=i_{U}^{*} \alpha_{2}$, then $i_{U}^{*} \omega_{M}\left(\alpha_{1}\right)=$ $\omega_{U}\left(i_{U}^{*} \alpha_{1}\right)=\omega_{U}\left(i_{U}^{*} \alpha_{2}\right)=i_{U}^{*} \omega_{M}\left(\alpha_{2}\right)$. In other words, $i_{U}^{*} \omega_{M}(\alpha)$ depends only on $i_{U}^{*} \alpha$. Next, we claim that $\omega_{M}(0)=0$. For we have just proved that if $p \in M$, the value of $\left.\omega_{M}(0)\right|_{p}$ can be computed by restricting to a neighborhood of $p$, which we may as well assume is a neighborhood of the origin in affine $m$-space $\mathbb{A}^{m}$. Then since the 1-form $\alpha=0$ is invariant under the linear group $G L_{m} \mathbb{R}$ acting on $\mathbb{A}^{m}$ fixing the origin, so too is the $q$-form $\left.\omega_{M}(0)\right|_{p=0 \in \mathbb{A}^{m}} \in \bigwedge^{q}\left(\mathbb{R}^{m}\right)^{*}$. Since $q>0$ the only $G L_{m} \mathbb{R}$-invariant $q$-form is zero.

Suppose that $\alpha_{1}, \alpha_{2} \in \Omega^{1}(M ; V)$ have identical $q$-jets at $p \in M$, so for some functions $f_{0}, f_{1}, \ldots, f_{q}$ which vanish at $p$ and some $\beta \in \Omega^{1}(M ; V)$, we have

$$
\alpha_{2}=\alpha_{1}+f_{0} f_{1} \cdots f_{q} \beta .
$$

Let $\tilde{\alpha}_{i}, \tilde{\beta}$ be the pullbacks of $\alpha_{i}, \beta$ to $M \times \mathbb{A}^{q+1}$, and let $t_{0}, t_{1}, \ldots, t_{q}$ be the standard coordinates on $\mathbb{A}^{q+1}$. Then $\alpha_{i}, f_{0} f_{1} \cdots f_{q} \beta$ are the pullbacks of $\tilde{\alpha}_{i}, t_{0} t_{1} \cdots t_{q} \tilde{\beta}$ via the map $i d_{M} \times\left(f_{0}, f_{1}, \ldots, f_{q}\right): M \rightarrow M \times \mathbb{A}^{q+1}$. Observe that this map sends $p$ 
to $(p, 0)$. By naturality it suffices to show that

$$
\left.\omega_{M \times \mathbb{A}^{q+1}}\left(\tilde{\alpha}_{2}\right)\right|_{(p, 0)} \quad \text { and }\left.\quad \omega_{M \times \mathbb{A}^{q+1}}\left(\tilde{\alpha}_{1}+t_{0} t_{1} \cdots t_{q} \tilde{\beta}\right)\right|_{(p, 0)}
$$

agree as $q$-forms on $T_{p} M \times \mathbb{R}^{q+1}$. Decomposing with respect to the standard basis of $\mathbb{R}^{q+1}$ and using multilinearity, we se 8 that it suffices to evaluate the $q$-forms (8.8) on $q$-vectors which have vanishing component along the $i$ th axis in $\mathbb{R}^{q+1}$ for some $i$. By naturality those evaluations can be made by restricting the forms $\omega\left(\tilde{\alpha}_{2}\right)$ and $\omega\left(\tilde{\alpha}_{1}+t_{0} t_{1} \cdots t_{q} \tilde{\beta}\right)$ to the submanifold $N_{i} \subset M \times \mathbb{A}^{q+1}$ defined by $t_{i}=0$. But these $q$-forms agree on $N_{i}$, since the 1 -forms $\tilde{\alpha}_{2}$ and $\tilde{\alpha}_{1}+t_{0} t_{1} \cdots t_{q} \tilde{\beta}$ restrict to equal 1-forms on $N_{i}$.

Lemma 8.6 implies that $\omega \in \Omega^{q}\left(\Omega^{1} \otimes V\right)$ is determined by its value at the origin of vector spaces $W \in \mathbf{M a n}$, and furthermore for $\alpha \in \Omega^{1}(W ; V)$ the value of $\omega_{W}(\alpha)$ at the origin of $W$ is computed by a map (of sets)

$$
\tilde{\omega}_{W}: J^{q}\left(W ; W^{*} \otimes V\right) \longrightarrow \bigwedge^{q} W^{*},
$$

where $J^{q}\left(W ; W^{*} \otimes V\right)$ is the finite-dimensional vector space of $q$-jets of elements of $\Omega^{1}(W ; V)$ at the origin. Furthermore, (8.9) is functorial in $W$, in particular for linear maps. Let Vect be the category of finite-dimensional real vector spaces and linear maps. Then

$$
\begin{aligned}
& W \longmapsto J^{q}\left(W ; W^{*} \otimes V\right) \\
& W \longmapsto \bigwedge^{q} W^{*}
\end{aligned}
$$

are polynomial functors Vect ${ }^{\text {op }} \rightarrow$ Vect, and the first is reduced. (See Appendix A for definitions.) The map (8.9) is a set-theoretic transformation between these two functors. The following statement is then a direct consequence 9 of Theorem A.26.

Lemma 8.11. The map $\tilde{\omega}_{W}$ is a polynomial of degree $\leq q$.

Next, use the isomorphism

$$
J^{q}\left(W ; W^{*} \otimes V\right) \cong \bigoplus_{j=0}^{q}\left(\operatorname{Sym}^{j} W^{*} \otimes W^{*} \otimes V\right)
$$

to write the polynomial $\tilde{\omega}_{W}(8.9)$ as a linear map

$$
T_{W}: \bigoplus_{i=0}^{q} \operatorname{Sym}^{i}\left(\bigoplus_{j=0}^{q}\left(\operatorname{Sym}^{j} W^{*} \otimes W^{*} \otimes V\right)\right) \longrightarrow \bigwedge^{q} W^{*} .
$$

Lemma 8.14 (Weyl). A nonzero $G L(W)$-invariant linear map $\bigotimes^{N} W^{*} \rightarrow \bigwedge^{q} W^{*}$ has $N=q$ and is a multiple of the antisymmetrization map.

Proof. That $N=q$ follows from invariance under the scaling subgroup $\mathbb{R}^{\times} \subset$ $G L(W)$. A theorem of Weyl asserts that $\otimes{ }^{q} W^{*}$ is a direct sum of irreducible representations of $G L(W)$ of multiplicity one. Antisymmetrization is an equivariant map $\otimes^{q} W^{*} \rightarrow \bigwedge^{q} W^{*}$, so any other map must be a multiple of it.

Lemma 8.15. $T_{W}$ factors through $\bigoplus_{i=0}^{q} \operatorname{Sym}^{i}\left(W^{*} \otimes V \oplus \bigwedge^{2} W^{*} \otimes V\right)$.

\footnotetext{
${ }^{8}$ Proposition A.9 is a general abstraction of this assertion.

${ }^{9}$ As Theorem A.26 applies to covariant functors, and (8.10) are contravariant, precompose with the contravariant functor $W \mapsto W^{*}$ before applying the theorem.
} 
Proof. Naturality implies that (8.13) is invariant under $G L(W)$. The domain of $T_{W}$ is a direct sum of quotients of subspaces $U$ of vector spaces of the form $\left(\otimes^{k} W^{*}\right) \otimes$ $\left(\otimes^{\ell} V\right)$ for some $k, \ell$. By Weyl's theorem any equivariant map must have $k=q$ and be a multiple of antisymmetrization $\bigotimes^{q} W^{*} \rightarrow \bigwedge^{q} W^{*}$ tensored with a multilinear form on $V$. The restriction of (8.13) to terms with $j \geq 2$ must vanish since for these terms the lift of (8.13) to a map $U \subset\left(\otimes^{k} W^{*}\right) \otimes\left(\otimes^{\ell} V\right) \rightarrow \bigwedge^{q} W^{*}$ has $k \geq 2$ and would be symmetric in at least two of the factors of $W^{*}$ in the domain, whereas antisymmetrization is completely antisymmetric. Similarly, for $j=1$ it must factor through the quotient $\bigwedge^{2} W^{*}$ of $W^{*} \otimes W^{*}$.

It follows from Lemma 8.15 that for each finite-dimensional real vector space $W$ there is a linear map

$$
L_{W}: \Omega^{\bullet}\left(\Omega^{1} \otimes V\right) \longrightarrow \operatorname{Hom}_{G L(W)}\left(\operatorname{Sym}\left(W^{*} \otimes V\right) \otimes \operatorname{Sym}\left(\bigwedge^{2} W^{*} \otimes V\right) ; \bigwedge^{\bullet} W^{*}\right),
$$

where Sym denotes the entire symmetric algebra. As $W$ varies, both of

$$
\begin{aligned}
& F_{1}: W \longmapsto \operatorname{Sym}\left(W^{*} \otimes V\right) \otimes \operatorname{Sym}\left(\bigwedge^{2} W^{*} \otimes V\right), \\
& F_{2}: W \longmapsto \bigwedge^{\bullet} W^{*}
\end{aligned}
$$

are polynomial functors Vect $^{\text {op }} \rightarrow$ Vect, and (8.16) defines a linear map $L$ from $\Omega^{\bullet}\left(\Omega^{1} \otimes V\right)$ to the vector space of natural transformations $F_{1} \rightarrow F_{2}$. Recall the linear map $\eta$ in (8.2).

Lemma 8.18. $L \circ \eta$ is injective.

Proof. The Koszul algebra $\operatorname{Kos}^{\bullet} V^{*}$ is doubly graded with homogeneous components

$$
\operatorname{Kos}^{p, q} V^{*}=\bigwedge^{p} V^{*} \otimes \operatorname{Sym}^{q} V^{*}, \quad p, q \in \mathbb{Z}^{\geq 0} .
$$

Identify $\operatorname{Kos}^{p, q} V^{*}$ with the space of multilinear maps $\phi: V^{\times(p+q)} \rightarrow \mathbb{R}$ which are skew-symmetric in the first $p$ arguments and symmetric in the last $q$ arguments. The codomain $A$ of $L$ is doubly graded with homogeneous components

$$
\begin{array}{r}
A^{p, q}(W)=\operatorname{Hom}_{G L(W)}\left(\operatorname{Sym}^{p}\left(W^{*} \otimes V\right) \otimes \operatorname{Sym}^{q}\left(\bigwedge^{2} W^{*} \otimes V\right) ; \bigwedge^{p+2 q} W^{*}\right), \\
p, q \in \mathbb{Z}^{\geq 0} .
\end{array}
$$

(Invariance under scaling dictates the degree $p+2 q$.) The map $L \circ \eta$ preserves the bigrading. Fix a bidegree $p, q$. The image of a multilinear map $\phi \in \operatorname{Kos}^{p, q} V^{*}$ under $L_{W} \circ \eta$ is the multilinear map

$$
\begin{aligned}
{\left[w_{1}^{*} \otimes v_{1}\right], \ldots,\left[w_{p}^{*} \otimes v_{p}\right],\left[\left(w_{p+1}^{*}\right.\right.} & \left.\left.\wedge w_{p+2}^{*}\right), v_{p+1}\right], \ldots,\left[\left(w_{p+2 q-1}^{*} \wedge w_{p+2 q}^{*}\right), v_{p+q}\right] \\
& \longmapsto \phi\left(v_{1}, \ldots, v_{p}, v_{p+1}, \ldots, v_{p+q}\right) w_{1}^{*} \wedge \cdots \wedge w_{p+2 q}^{*}
\end{aligned}
$$

where $v_{i} \in V, w_{i}^{*} \in W^{*}$. To prove that $L \circ \eta$ is injective in bidegree $p, q$ choose $\operatorname{dim} W=p+2 q$, fix a basis $w_{1}^{*}, \ldots, w_{p+2 q}^{*}$ of $W^{*}$, and evaluate (8.21) on all $v_{i} \in V$ to see that if $\left(L_{W} \circ \eta\right) \phi=0$, then $\phi=0$. 
Proof of Theorem 8.1. Lemma 8.6 and the subsequent arguments prove that $L$ is injective. To prove Theorem 8.1, which asserts that $\eta$ is an isomorphism, it suffices now to show that $L \circ \eta$ is surjective. We again fix a bidegree $p, q$ and a vector space $W$. Let $h \in A^{p, q}(W)$ (see (8.20) $)$. Then $h$ lifts to an element $\tilde{h}$ of

$$
\begin{aligned}
\operatorname{Hom}_{G L(W)}\left(\bigotimes^{p}\left(W^{*} \otimes V\right) \otimes \bigotimes^{q}\left(\bigotimes^{2} W^{*} \otimes V\right) ; \bigwedge^{p+2 q} W^{*}\right) \\
\cong \operatorname{Hom}_{G L(W)}\left(\bigotimes^{p+2 q}\left(W^{*}\right) \otimes \bigotimes^{p+q}(V) ; \bigwedge^{p+2 q} W^{*}\right)
\end{aligned}
$$

By Lemma 8.14, $\tilde{h}$ must have the form

$$
\begin{aligned}
{\left[w_{1}^{*} \otimes v_{1}\right], \ldots,\left[w_{p}^{*} \otimes v_{p}\right],\left[\left(w_{p+1}^{*} \wedge w_{p+2}^{*}\right),\right.} & \left.v_{p+1}\right], \ldots,\left[\left(w_{p+2 q-1}^{*} \wedge w_{p+2 q}^{*}\right), v_{p+q}\right] \\
& \longmapsto \phi\left(v_{1}, \ldots, v_{p+q}\right) w_{1}^{*} \wedge \cdots \wedge w_{p+2 q}^{*},
\end{aligned}
$$

for some $\phi \in \bigotimes^{p+q}\left(V^{*}\right)$. The fact that $\tilde{h}$ factors through $A^{p, q}(W)$ in 8.20 implies that $\phi$ is skew-symmetric in the first $p$ variables and symmetric in the last $q$, so $h$ is in the image of $\operatorname{Kos}^{p, q} V^{*} \rightarrow A^{p, q}(W)$.

The following is a slight generalization of Theorem 7.28 (i).

Corollary 8.24. Let $V$ be a finite-dimensional real vector space and $Y$ a smooth manifold. Then the de Rham complex of $Y \times\left(\Omega^{1} \otimes V\right)$ is $\Omega\left(Y ; \operatorname{Kos} V^{*}\right)^{\bullet}$ with differential the sum of the de Rham differential on $Y$ and the Koszul differential (7.17).

The de Rham complex of a Cartesian product of manifolds is a completed tensor product of their individual de Rham complexes, and this corollary would follow from that statement for generalized manifolds (where there is no completion since the de Rham complex of $\Omega^{1} \otimes V$ is finite dimensional).

Proof. We indicate the modifications to the proof of Theorem 8.1 to accommodate the factor of $Y$. Define a homomorphism

$$
\eta_{Y}: \Omega\left(Y ; \operatorname{Kos} V^{*}\right)^{\bullet} \longrightarrow \Omega^{\bullet}\left(Y \times \Omega^{1} \otimes V\right)
$$

as the composition

$$
\Omega\left(Y ; \operatorname{Kos} V^{*}\right)^{\bullet} \stackrel{\eta}{\longrightarrow} \Omega\left(Y ; \Omega\left(\Omega^{1} \otimes V\right)\right) \stackrel{\wedge}{\longrightarrow} \Omega\left(Y \times \Omega^{1} \otimes V\right) .
$$

We must prove that $\eta_{Y}$ is an isomorphism. In Lemma 8.6 for each test manifold $M$ we have a pair $\phi, \alpha$ consisting of a map $\phi: M \rightarrow Y$ and a 1-form $\alpha \in \Omega^{1}(M ; V)$; now $\omega_{M}=\omega_{M}(\alpha, \phi)$. The locality argument implies that to compute $i_{U}^{*} \omega_{M}(\alpha, \phi)$ for $U \subset M$ a small open set containing $p \in M$, we can replace $Y$ by an open set in a vector space $V^{\prime}$. In the paragraph containing (8.7) define $\tilde{\phi}: M \times \mathbb{A}^{q+1} \rightarrow V^{\prime}$ as the composition $\phi \circ \pi_{1}$. If $\phi_{1}, \phi_{2}$ have the same $q$-jet at $p$, then $\tilde{\phi}_{2}=\tilde{\phi}_{1}+t_{0} t_{1} \cdots t_{q} \tilde{\psi}$ for some $\psi: M \rightarrow V^{\prime}$. A similar argument to that surrounding (8.8) shows that $\left.\omega_{M}(\alpha, \phi)\right|_{p}$ only depends on the $q$-jet of $\phi$ at $p$, and so we take $V^{\prime}=T_{y} Y$, where $y=\phi(p)$. Then in (8.9) we replace $J^{q}\left(W ; W^{*} \otimes V\right)$ with the $q$-jets of elements of $\Omega^{1}(W ; V) \times \operatorname{Map}\left(W ; T_{y} Y\right)$ at the origin of $W$. Lemma 8.11 is unchanged. The isomorphism (8.12) is replaced by

$$
J^{q}\left(W ; W^{*} \otimes V \oplus T_{y} Y\right) \cong \bigoplus_{j=0}^{q}\left(\operatorname{Sym}^{j} W^{*} \otimes\left(W^{*} \otimes V \oplus T_{y} Y\right)\right) .
$$


In the statement of Lemma 8.15 there is an extra term $W^{*} \otimes T_{y} Y$ under $\mathrm{Sym}^{i}$ for each $i$, and (8.16) is now

$$
\begin{aligned}
L_{y, W} & : \Omega^{\bullet}\left(Y \times \Omega^{1} \otimes V\right) \\
& \longrightarrow \operatorname{Hom}_{G L(W)}\left(\operatorname{Sym}\left(W^{*} \otimes V \oplus \bigwedge^{2} W^{*} \otimes V \oplus W^{*} \otimes T_{y} Y\right) ; \bigwedge^{\bullet} W^{*}\right) .
\end{aligned}
$$

The composite $L_{y, W} \circ \eta_{Y}$ preserves a triple grading:

$$
\begin{aligned}
& \Omega^{r}\left(Y ; \operatorname{Kos}^{p, q} V^{*}\right) \\
& \longrightarrow \operatorname{Hom}_{G L(W)}\left(\operatorname{Sym}^{p}\left(W^{*} \otimes V\right) \otimes \operatorname{Sym}^{q}\left(\bigwedge^{2} W^{*} \otimes V\right)\right. \\
& \left.\otimes \operatorname{Sym}^{r}\left(W^{*} \otimes T_{y} Y\right) ; \bigwedge^{p+2 q+r} W^{*}\right) .
\end{aligned}
$$

The proofs that $L_{y} \circ \eta_{Y}$ is injective and surjective are similar to those above, so are omitted.

Proof of Lemma 7.25. We use (7.11). So if $\alpha: M \rightarrow \Omega^{1} \otimes \mathfrak{g}$ is a $\mathfrak{g}$-valued 1-form on a test manifold $M$, and $\lambda \in \mathfrak{g}^{*}$, then by (8.3) the pullback of $\eta(\alpha)$ to $M$ is the scalar 1-form $\langle\alpha, \lambda\rangle \in \Omega^{1}(M)$. The action (5.22) of $G$ gives the 1-form $\left\langle\theta+\operatorname{Ad}_{g^{-1}} \alpha, \lambda\right\rangle \in$ $\Omega^{1}(G \times M)$, and the contraction with $\xi \in G$ along $\{e\} \times M$ is $\langle\xi, \lambda\rangle$, as claimed in the first formula of (7.26). By (8.5) the functional $\lambda \in \mathfrak{g}^{*}$ also determines, via $\eta$, a scalar 2-form $\langle d \alpha, \lambda\rangle \in \Omega^{2}(M)$, and the action of $G$ produces the 2 -form

$$
\left\langle\frac{1}{2}[\theta, \theta]+\operatorname{Ad}_{d\left(g^{-1}\right)} \wedge \alpha+\operatorname{Ad}_{g^{-1}} d \alpha, \lambda\right\rangle \in \Omega^{2}(G \times M) .
$$

Only the second term contributes to the restriction to $\{e\} \times M$ of the contraction with $\xi$, which equals $\left\langle-\operatorname{Ad}_{\xi} \alpha, \lambda\right\rangle=\left\langle\alpha,-\operatorname{Ad}_{\xi}^{*} \lambda\right\rangle \in \Omega^{1}(M)$, the 1-form corresponding to $-\operatorname{Ad}_{\xi}^{*} \lambda \in \mathfrak{g}^{*}$. This proves the second line of (7.26).

Proof of Theorem 7.20. By Proposition 5.24 and the fact that Definition 7.1 takes place in the homotopy category, de Rham complex of $B_{\nabla} G$ equals that of $B_{\nabla}^{\text {triv }} G$. Now $B_{\nabla}^{\text {triv }} G$ is the simplicial sheaf (5.21) which represents the action of $G$ on $\Omega^{1} \otimes \mathfrak{g}$. By Proposition 7.5 and Proposition 7.14 its de Rham complex is the basic subcomplex of $\Omega^{\bullet}\left(\Omega^{1} \otimes \mathfrak{g}\right)$. By Theorem 7.19 the latter is the Weil algebra (Kos $\mathfrak{g}^{*}, d_{K}$ ). Thus we are reduced to computing the basic subcomplex of the Weil algebra, which is standard.

Following $\mathrm{MQ}, \S 5]$, choose a basis $\left\{e_{i}\right\}$ of $\mathfrak{g}$ and corresponding dual basis $\left\{e^{i}\right\}$ of $\mathfrak{g}^{*}$. Let $\iota_{i}, \theta^{i}$ be interior multiplication by $e_{i}$ and exterior multiplication by $e^{i}$, respectively, on $\Lambda^{\bullet} \mathfrak{g}^{*}$. Then it is easy to see that $\prod_{i}\left(1-\theta^{i} \iota_{i}\right)$ is projection onto the horizontal elements of $\wedge^{\bullet} \mathfrak{g}^{*}$ - those which satisfy Definition 7.13)(ii) — and that the image of this projector is in fact $\bigwedge^{0} \mathfrak{g}^{*} \subset \bigwedge^{\bullet} \mathfrak{g}^{*}$. Now $d \theta^{i} \subset \operatorname{Sym}^{1} \mathfrak{g}^{*} \subset \operatorname{Kos}^{\bullet} \mathfrak{g}^{*}$, and we set

$$
\Omega^{i}=d \theta^{i}+\frac{1}{2} f_{j k}^{i} \theta^{j} \wedge \theta^{k},
$$

where $\left[e_{j}, e_{k}\right]=f_{j k}^{i} e_{i}$ and we use the summation convention. A short computation from (7.26) shows that $\iota_{\ell} \Omega^{i}=0$ for all $i, \ell$. By a change of basis we may identify the Weil algebra as the exterior algebra on the span of $\left\{\theta^{i}\right\}$ tensor the symmetric algebra on the span of $\left\{\Omega^{i}\right\}$. It follows that the horizontal elements of the Weil algebra form the subspace $\bigwedge^{0} \mathfrak{g}^{*} \otimes \operatorname{Sym} \bullet \mathfrak{g}^{*}$, where the second factor is the symmetric 
algebra on the span of $\left\{\Omega^{i}\right\}$. Therefore, the basic subalgebra of the Weil algebra are the $G$-invariants in that symmetric algebra, as claimed.

Remark 8.32. The computation of the basic subcomplex of the Weil algebra is a special case of the computation of the basic subcomplex in Theorem 7.28 (ii), which results in the Cartan model for equivariant de Rham cohomology; see [MQ, §5], GS, §4].

\section{Appendix A. Transformations of POLYNomial FUnCtors}

Polynomial functors are intimately related to Schur's representation theory of the symmetric group; for example, see [Mac, §I, Appendix]. In this appendix we prove that every set-theoretic natural transformation of polynomial functors is polynomial.

Let Vect be the category of finite-dimensional vector spaces over $10 \mathbb{R}$.

Definition A.1. A functor $F$ : Vect $\rightarrow$ Vect is polynomial of degree $d$ if for every $V$ and every $f_{1}, \ldots, f_{n} \in$ End $V$, the map

$$
F\left(\lambda_{1} f_{1}+\cdots+\lambda_{n} f_{n}\right)
$$

is a polynomial of degree $d$ in $\lambda_{1}, \ldots, \lambda_{n}$ with coefficients in $\operatorname{End}(F(V))$. A functor $F$ is homogeneous of degree $d$ if the above polynomial is homogeneous of degree $d$.

To keep the language simple we make the convention that a polynomial of degree $d$ might also be a polynomial of lower degree.

Suppose that $F$ is polynomial functor of degree $d$, and write

$$
F\left(\lambda \operatorname{id}_{V}\right)=\sum_{i=0}^{d} \lambda^{i} \mathbf{e}_{i}(V), \quad \lambda \in k, \quad \mathbf{e}_{i}(V) \in \operatorname{End}(F(V)) .
$$

Using

$$
F\left(\lambda_{1} \lambda_{2} \text { id }\right)=F\left(\left(\lambda_{1} \text { id }\right) \circ\left(\lambda_{2} \text { id }\right)\right)=F\left(\lambda_{1} \text { id }\right) \circ F\left(\lambda_{2}(\text { id })\right),
$$

one easily checks that the $\mathbf{e}_{i}(V): F(V) \rightarrow F(V)$ are orthogonal idempotents. Write $F_{i}=\mathbf{e}_{i} F$. Then $F_{i}$ is homogeneous of degree $i$, and $F=\bigoplus F_{i}$.

Here is a useful fact about polynomial functors. For a subset $I \subset\{1, \ldots, n\}$, let

$$
V^{I}=\left\{\left(v_{1}, \ldots, v_{n}\right) \in V^{n}: v_{i}=0, i \neq I\right\}
$$

be the ' $I$-axis', and let $\epsilon_{I}: V^{n} \rightarrow V^{n}$ be the projection operator to $V^{I}$. We write $\epsilon_{i}$ instead of $\epsilon_{\{i\}}$, so that

$$
\begin{aligned}
\epsilon_{i}\left(v_{1}, \ldots, v_{n}\right) & =\left(0, \ldots, v_{i}, \ldots, 0\right), \\
\epsilon_{I} & =\sum_{i \in I} \epsilon_{i}
\end{aligned}
$$

and

$$
\operatorname{id}_{V^{n}}=\epsilon_{1}+\cdots+\epsilon_{n} .
$$

Write $|I|$ for the number of elements of $I$.

\footnotetext{
${ }^{10}$ With the exception of the proof of Lemma A.18 the arguments in this appendix work over any field of characteristic zero.
} 
Proposition A.9. Suppose that $F:$ Vect $\rightarrow$ Vect is a polynomial functor of degree $d$, and let $n>d$. For every nonzero $x \in F\left(V^{n}\right)$ there is a subset $I \subset\{1, \ldots, n\}$ with $|I| \leq d$ and $F\left(\epsilon_{I}\right)(x) \neq 0$. Equivalently, the product of the restriction maps

$$
\prod_{|I|=d} F\left(\epsilon_{I}\right): F\left(V^{n}\right) \rightarrow \prod_{|I|=d} F\left(V^{I}\right)
$$

is a monomorphism.

Proof. Since $F$ is polynomial, we have

$$
F\left(\lambda_{1} \epsilon_{1}+\cdots+\lambda_{n} \epsilon_{n}\right)=\sum_{J} \lambda^{J} e_{J}
$$

in which $J=\left(j_{1}, \ldots, j_{n}\right), \lambda^{J}=\lambda_{1}^{j_{1}} \cdots \lambda_{n}^{j_{n}}$, and $e_{J}$ is an endomorphism of $F\left(V^{n}\right)$. As above, the $e_{J}$ are orthogonal idempotents. Setting all of the $\lambda_{i}=1$ and using (A.8), one sees that

$$
\sum_{J} e_{J}=\mathrm{id}
$$

Let $x$ be a nonzero element of $F\left(V^{n}\right)$. Apply (A.12) to $x$ to conclude that $e_{J}(x) \neq 0$ for some $J$. Since the degree of $F$ is $d$, at most $d$ of the $j_{i} \in J$ are nonzero. Let $I=\left\{i \mid j_{i} \neq 0\right\}$. Set

$$
\lambda_{i}= \begin{cases}1, & i \in I \\ 0, & i \notin I\end{cases}
$$

in (A.11) to deduce

$$
e_{J}\left(F\left(\epsilon_{I}\right)(x)\right)=e_{J}\left(e_{J}(x)+\cdots\right)=e_{J}(x) \neq 0,
$$

whence $F\left(\epsilon_{I}\right)(x) \neq 0$.

Definition A.15. Suppose that $V$ and $W$ are real vector spaces. A function $f: V \rightarrow W$ is polynomial (of degree $d$ ) if for every set $v_{1}, \ldots, v_{n}$ of elements of $V$, the map

$$
f\left(\lambda_{1} v_{1}+\cdots+\lambda_{n} v_{n}\right)
$$

is a polynomial in the $\lambda_{i}$ (of degree $d$ ) with coefficients in $W$; i.e., there exists a polynomial $g\left(t_{1}, \ldots, t_{n}\right) \in W \otimes_{\mathbb{R}} \mathbb{R}\left[t_{1}, \ldots, t_{n}\right]$ with the property that for all $\lambda_{1}, \ldots, \lambda_{n} \in \mathbb{R}$, and all $v_{1}, \ldots, v_{n} \in V$

$$
f\left(\lambda_{1} v_{1}+\cdots+\lambda_{n} v_{n}\right)=g\left(\lambda_{1}, \ldots, \lambda_{k}\right) .
$$

The following lemma gives a useful criterion for a map to be polynomial.

Lemma A.18. Let $k>0$ be an integer. Let $f: V \rightarrow W$ be a map, and assume that for every finite set $v_{1}, \ldots, v_{n}$ of elements of $V$ the map

$$
f\left(\lambda_{1}^{k} v_{1}+\cdots+\lambda_{n}^{k} v_{n}\right)
$$

is a polynomial in the $\lambda_{i}$ (of degree $k d$ ) with coefficients in $W$. Then $f$ is a polynomial map of degree $d$. 
Proof. By choosing bases of $V$ and $W$ we immediately reduce to the case $f: \mathbb{R}^{m} \rightarrow$ $\mathbb{R}$ for $m=\operatorname{dim} V$. The hypothesis implies that

$$
f\left(\lambda_{1}^{k}, \ldots, \lambda_{m}^{k}\right)=\sum_{r_{1}, \ldots, r_{m}} a_{r_{1} \cdots r_{m}} \lambda_{1}^{r_{1}} \cdots \lambda_{m}^{r_{m}}
$$

is a polynomial of degree $k d$ in $\lambda_{1}, \ldots, \lambda_{m} \in \mathbb{R}$ and

$$
f\left(\mu_{1}^{k}-\nu_{1}^{k}, \ldots, \mu_{m}^{k}-\nu_{m}^{k}\right)=\sum_{\substack{p_{1}, \ldots, p_{m} \\ q_{1}, \ldots, q_{m}}} b_{p_{1} \cdots p_{m} q_{1} \cdots q_{m}} \mu_{1}^{p_{1}} \cdots \mu_{m}^{p_{m}} \nu_{1}^{q_{1}} \cdots \nu_{m}^{q_{m}}
$$

is a polynomial of degree $k d$ in $\mu_{1}, \ldots, \mu_{m} \nu_{1}, \ldots, \nu_{m} \in \mathbb{R}$. For $\left|\mu_{i}\right| \geq\left|\nu_{i}\right|$ set $\lambda_{i}=\left(\mu_{i}^{k}-\nu_{i}^{k}\right)^{1 / k}$. Comparing (A.20) and (A.21), we find for each $r_{1}, \ldots, r_{m}$ that $(\mathrm{A} .22)$

$a_{r_{1} \cdots r_{m}}\left(\mu_{1}^{k}-\nu_{1}^{k}\right)^{r_{1} / k} \cdots\left(\mu_{m}^{k}-\nu_{m}^{k}\right)^{r_{m} / k}=\sum_{p_{i}+q_{i}=r_{i}} b_{p_{1} \cdots p_{m} q_{1} \cdots q_{m}} \mu_{1}^{p_{1}} \cdots \mu_{m}^{p_{m}} \nu_{1}^{q_{1}} \cdots \nu_{m}^{q_{m}}$.

If $a_{r_{1} \cdots r_{m}} \neq 0$ we conclude that

$$
\left(\mu_{1}^{k}-\nu_{1}^{k}\right)^{r_{1} / k} \cdots\left(\mu_{m}^{k}-\nu_{m}^{k}\right)^{r_{m} / k}
$$

is a homogeneous polynomial in $\mu_{1}, \ldots, \mu_{m}, \nu_{1}, \ldots, \nu_{m}$ on the region where $\left|\mu_{i}\right| \geq$ $\left|\nu_{i}\right|$ for all $i$. Apply Taylor's theorem at any point of that region to deduce that each of $r_{1}, \ldots, r_{m}$ is divisible by $k$ (else A.23 is not a polynomial). It follows from A.22 that $b_{p_{1} \cdots p_{m} q_{1} \cdots q_{m}}$ vanishes unless each of $p_{1}, \ldots, p_{m}, q_{1}, \ldots, q_{m}$ is divisible by $k$. If $k$ is odd, the vanishing now implies from (A.20) that $f\left(x_{1}, \ldots, x_{m}\right)$ is a polynomial of degree $d$ in $x_{1}, \ldots, x_{m}$, but if $k$ is even, we only deduce this on the region where all $x_{i} \geq 0$. From (A.21) we see that $f\left(y_{1}-z_{1}, \ldots, y_{m}-z_{m}\right)$ is (i) a polynomial of degree $d$ in $y_{1}, \ldots, y_{m}, z_{1}, \ldots, z_{m}$ and (ii) a polynomial of degree $d$ in $y_{1}-z_{1}, \ldots, y_{m}-z_{m}$ in the region where all $y_{i} \geq z_{i}$. From these two facts it follows that (ii) holds for all $y_{1}, \ldots, y_{m}, z_{1}, \ldots z_{m}$, whence $f\left(x_{1}, \ldots, x_{m}\right)$ is a polynomial of degree $d$ in $x_{1}, \ldots, x_{m}$.

Definition A.24. A polynomial functor

$$
F=\bigoplus_{i \geq 0} F_{i}
$$

is reduced if $F_{0}=0$.

We now prove the main result of this appendix.

Theorem A.26. Let $F$ and $G$ be polynomial functors over $\mathbb{R}$, and suppose that $F$ is reduced. Any set-theoretic transformation $T: F \rightarrow G$ is polynomial. If $G$ is polynomial of degree $d$, then $T$ is polynomial of degree $d$.

A set-theoretic transformation of functors $T: F \rightarrow G$ is a natural transformation of the underlying set-valued functors. In other words, the map $T(V): F(V) \rightarrow G(V)$, $V \in$ Vect, is a map of sets (which is not assumed to be linear).

Proof. Let $V$ be a vector space, $v_{1}, \ldots, v_{n} \in F(V)$. We wish to show that

$$
T_{V}\left(\lambda_{1} v_{1}+\cdots+\lambda_{n} v_{n}\right) \in G(V)
$$

is a polynomial function of $\lambda_{i} \in k$. For each $i$, write

$$
v_{i}=\sum v_{i}^{(j)}
$$


with $v_{i}^{(j)} \in F_{j}(V)$. Then since

$$
\sum \lambda_{i} v_{i}=\sum \lambda_{i} v_{i}^{(j)}
$$

we might as well assume from the outset that each $v_{i}$ is homogeneous in the sense that $v_{i} \in F_{k_{i}}(V)$ for some $k_{i}$. Since $F$ is reduced, none of the $k_{i}$ is zero. We may therefore choose an integer $k$ which is divisible by all the $k_{i}$. We will show that

$$
T_{V}\left(\lambda_{1}^{k} v_{1}+\cdots+\lambda_{n}^{k} v_{n}\right) \in G(V)
$$

is a polynomial function of the $\lambda_{i}$, of degree $k d$ if $G$ has degree $d$. By Lemma A.18 this implies that $T_{V}$ is polynomial of degree $d$.

Consider the following commutative diagram, in which $\Sigma: V^{n} \rightarrow V$ is the sum map:

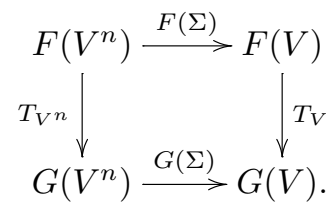

Let $j_{i}: V \rightarrow V^{n}$ be the inclusion of the $i$ th subspace $0 \oplus \cdots \oplus V \oplus \cdots \oplus 0 \subset V^{n}$. Under the top arrow $F(\Sigma)$, the vector

$$
\lambda_{1}^{k} F\left(j_{1}\right)\left(v_{1}\right)+\cdots+\lambda_{n}^{k} F\left(j_{n}\right)\left(v_{n}\right) \in F\left(V^{n}\right)
$$

maps to $\lambda_{1}^{k} v_{1}+\cdots+\lambda_{n}^{k} v_{n} \in F(V)$. By commutativity of the diagram, it therefore suffices to show that

$$
T_{V^{n}}\left(\lambda_{1}^{k} F\left(j_{1}\right)\left(v_{1}\right)+\cdots+\lambda_{n}^{k} F\left(j_{n}\right)\left(v_{n}\right)\right) \in G\left(V^{n}\right)
$$

is a polynomial function of the $\lambda_{i}$ with coefficients in $G\left(V^{n}\right)$.

Let $f: V^{n} \rightarrow V^{n}$ be the map

$$
f=\lambda_{1}^{k / k_{1}} \epsilon_{1}+\cdots+\lambda_{n}^{k / k_{n}} \epsilon_{n},
$$

where $\epsilon_{i}\left(v_{1}, \ldots, v_{n}\right)=\left(0, \ldots, v_{i}, \ldots, 0\right)$. Since $G$ is polynomial, it follows that that $G(f)$ is a polynomial in $\lambda_{i}^{k / k_{i}}$ (and hence in the $\lambda_{i}$ ) with coefficients in $\operatorname{End}\left(G\left(V^{n}\right)\right)$. Now follow the element

$$
x=F\left(j_{1}\right)\left(v_{1}\right)+\cdots+F\left(j_{n}\right)\left(v_{n}\right) \in F\left(V^{n}\right)
$$

around the commutative diagram

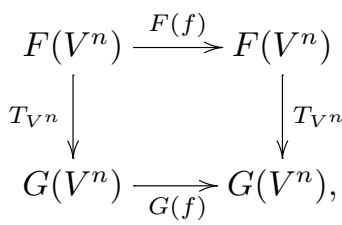

starting in the upper left corner. It is sent by the top horizontal arrow to

$$
\lambda_{1}^{k} F\left(j_{1}\right)\left(v_{1}\right)+\cdots+\lambda_{n}^{k} F\left(j_{n}\right)\left(v_{n}\right) \in F\left(V^{n}\right),
$$

which in turn is sent by the right vertical arrow to A.33). Under the left vertical arrow $x$ is sent to $T_{V^{n}}(x)$ which, since $G(f)$ is a polynomial endomorphism (in the $\lambda_{i}$ ), is sent by the bottom horizontal arrow to a polynomial in the $\lambda_{i}$ with coefficients in $G\left(V^{n}\right)$. If $G$ is polynomial of degree $d$, then $G(f)$ is a polynomial 
in $\lambda_{1}^{k / k_{1}}, \ldots, \lambda_{n}^{k / k_{n}}$ of degree $d$, so a polynomial in $\lambda_{1}, \ldots, \lambda_{n}$ of degree $k d$. This completes the proof.

\section{ABOUT THE AUTHORS}

Longtime collaborators, the authors share a common interest in geometry, topology, and physics.

\section{REFERENCES}

[AB] M. F. Atiyah and R. Bott, The moment map and equivariant cohomology, Topology 23 (1984), no. 1, 1-28, DOI 10.1016/0040-9383(84)90021-1. MR721448 (85e:58041)

[ABP] M. Atiyah, R. Bott, and V. K. Patodi, On the heat equation and the index theorem, Invent. Math. 19 (1973), 279-330. MR0650828 (58 \#31287)

[Bo1] A. K. Bousfield, The localization of spaces with respect to homology, Topology 14 (1975), 133-150. MR0380779 (52 \#1676)

[Bo2] A. K. Bousfield, The localization of spectra with respect to homology, Topology 18 (1979), no. 4, 257-281, DOI 10.1016/0040-9383(79)90018-1. MR.551009 (80m:55006)

[Br] Kenneth S. Brown, Abstract homotopy theory and generalized sheaf cohomology, Trans. Amer. Math. Soc. 186 (1973), 419-458. MR0341469 (49 \#6220)

[Bu] Ulrich Bunke, Differential cohomology, arXiv:1208.3961.

[C1] Henri Cartan, Notions d'algèbre différentielle; application aux groupes de Lie et aux variétés où opère un groupe de Lie, Colloque de topologie (espaces fibrés), Bruxelles, 1950, Georges Thone, Liège, 1951, pp. 15-27 (French). Reprinted in [GS]. MR.0042426 $(13,107 \mathrm{e})$

[C2] Henri Cartan, La transgression dans un groupe de Lie et dans un espace fibré principal, Colloque de topologie (espaces fibrés), Bruxelles, 1950, Georges Thone, Liège, 1951, pp. 57-71. Reprinted in GS.

[ChS] Jeff Cheeger and James Simons, Differential characters and geometric invariants, Geometry and topology (College Park, Md., 1983/84), Lecture Notes in Math., vol. 1167, Springer, Berlin, 1985, pp. 50-80, DOI 10.1007/BFb0075216. MR827262 (87g:53059)

[CS] Shiing Shen Chern and James Simons, Characteristic forms and geometric invariants, Ann. of Math. (2) 99 (1974), 48-69. MR0353327 (50 \#5811)

[Cu] Edward B. Curtis, Simplicial homotopy theory, Advances in Math. 6 (1971), 107-209 (1971). MR0279808 (43 \#5529)

[D] Pierre Deligne, Équations différentielles à points singuliers réguliers, Lecture Notes in Mathematics, Vol. 163, Springer-Verlag, Berlin, 1970 (French). MR0417174 (54 \#5232)

[DHKS] William G. Dwyer, Philip S. Hirschhorn, Daniel M. Kan, and Jeffrey H. Smith, Homotopy limit functors on model categories and homotopical categories, Mathematical Surveys and Monographs, vol. 113, American Mathematical Society, Providence, RI, 2004. MR 2102294 (2005k:18027)

[DHZ] Johan Dupont, Richard Hain, and Steven Zucker, Regulators and characteristic classes of flat bundles, The arithmetic and geometry of algebraic cycles (Banff, AB, 1998), CRM Proc. Lecture Notes, vol. 24, Amer. Math. Soc., Providence, RI, 2000, pp. 4792. MR 1736876 (2001c:14042)

[DS] W. G. Dwyer and J. Spaliński, Homotopy theories and model categories, Handbook of Algebraic Topology, North-Holland, Amsterdam, 1995, pp. 73-126, DOI 10.1016/B978044481779-2/50003-1. MR1361887 (96h:55014)

[E] Charles Ehresmann, Les connexions infinitésimales dans un espace fibré différentiable, Colloque de topologie (espaces fibrés), Bruxelles, 1950, Georges Thone, Liège, 1951, pp. 29-55 (French). MR0042768 (13,159e)

[Fr] Greg Friedman, Survey article: an elementary illustrated introduction to simplicial sets, Rocky Mountain J. Math. 42 (2012), no. 2, 353-423, DOI 10.1216/RMJ-2012-42-2-353. MR 2915498

[FSS] Domenico Fiorenza, Urs Schreiber, and Jim Stasheff, Cech cocycles for differential characteristic classes - An $\infty$-Lie theoretic construction, arXiv:1011.4735

$[\mathrm{FT}]$ Daniel S. Freed and Constantin Teleman, Relative quantum field theory, arXiv:1212.1692 
[G] Peter B. Gilkey, Invariance theory, the heat equation, and the Atiyah-Singer index theorem, 2nd ed., Studies in Advanced Mathematics, CRC Press, Boca Raton, FL, 1995. MR 1396308 (98b:58156)

[GJ] Paul G. Goerss and John F. Jardine, Simplicial homotopy theory, Progress in Mathematics, vol. 174, Birkhäuser Verlag, Basel, 1999. MR.1711612 (2001d:55012)

[GS] Victor W. Guillemin and Shlomo Sternberg, Supersymmetry and equivariant de Rham theory, Mathematics Past and Present, Springer-Verlag, Berlin, 1999. With an appendix containing two reprints by Henri Cartan [MR0042426 (13,107e); MR0042427 (13,107f)]. MR.1689252 (2001i:53140)

[HS] M. J. Hopkins and I. M. Singer, Quadratic functions in geometry, topology, and M-theory, J. Differential Geom. 70 (2005), no. 3, 329-452. MR2192936(2007b:53052)

[J] J. F. Jardine, The Verdier hypercovering theorem, Canad. Math. Bull. 55 (2012), no. 2, 319-328, DOI 10.4153/CMB-2011-093-x. MR2957248

[Joy] Dominic Joyce, D-manifolds, d-orbifolds and derived differential geometry: a detailed summary, arXiv: 1208.4948.

[K] Felix Klein, Vergleichende Betrachtungen über neuere geometrische Forschungen, Math. Ann. 43 (1893), no. 1, 63-100, DOI 10.1007/BF01446615 (German). MR.1510801

[Ku] Shrawan Kumar, A remark on universal connections, Math. Ann. 260 (1982), no. 4, 453-462, DOI 10.1007/BF01457024. MR670193 (84d:53028)

[Ma] J. Peter May, Simplicial objects in algebraic topology, Van Nostrand Mathematical Studies, No. 11, D. Van Nostrand Co., Inc., Princeton, N.J.-Toronto, Ont.-London, 1967. MR $0222892(36$ \#5942)

[Mac] I. G. Macdonald, Symmetric functions and Hall polynomials, 2nd ed., Oxford Mathematical Monographs, The Clarendon Press Oxford University Press, New York, 1995. With contributions by A. Zelevinsky; Oxford Science Publications. MR.1354144 (96h:05207)

[Mi] John Milnor, The geometric realization of a semi-simplicial complex, Ann. of Math. (2) 65 (1957), 357-362. MR.0084138 (18,815d)

[MM] Saunders Mac Lane and Ieke Moerdijk, Sheaves in geometry and logic, Universitext, Springer-Verlag, New York, 1994. A first introduction to topos theory; Corrected reprint of the 1992 edition. MR1300636 (96c:03119)

[MP] M. Mostow and J. Perchik, Notes on Gelfand-Fuks cohomology and characteristic classes (Lectures by R. Bott), Proceedings of the eleventh annual holiday symposium, New Mexico State University, 1973, pp. 1-126. reprinted in Collected Papers of Raoul Bott, vol. 3, Birkhauser, Boston, 1995, pp. 288-356.

[MQ] Varghese Mathai and Daniel Quillen, Superconnections, Thom classes, and equivariant differential forms, Topology 25 (1986), no. 1, 85-110, DOI 10.1016/0040-9383(86)900078. MR836726 (87k:58006)

[MS] John W. Milnor and James D. Stasheff, Characteristic classes, Princeton University Press, Princeton, N. J., 1974. Annals of Mathematics Studies, No. 76. MR0440554 (55 \#13428)

[NR] M. S. Narasimhan and S. Ramanan, Existence of universal connections. II, Amer. J. Math. 85 (1963), 223-231. MR0151923 (27 \#1904)

[Q1] Daniel G. Quillen, Homotopical algebra, Lecture Notes in Mathematics, No. 43, SpringerVerlag, Berlin, 1967. MR0223432 (36 \#6480)

[Q2] Daniel Quillen, Rational homotopy theory, Ann. of Math. (2) 90 (1969), 205-295. MR0258031 (41 \#2678)

[Q3] Daniel Quillen, Superconnections and the Chern character, Topology 24 (1985), no. 1, 89-95, DOI 10.1016/0040-9383(85)90047-3. MR790678 (86m:58010)

[R] T. R. Ramadas, On the space of maps inducing isomorphic connections, Ann. Inst. Fourier (Grenoble) 32 (1982), no. 1, viii, 263-276 (English, with French summary). MR 658951 (84h:53038)

[S] Graeme Segal, Classifying spaces and spectral sequences, Inst. Hautes Études Sci. Publ. Math. 34 (1968), 105-112. MR0232393(38 \#718)

[Sch] Roger Schlafly, Universal connections, Invent. Math. 59 (1980), no. 1, 59-65, DOI 10.1007/BF01390314. MR.575081 (81f:53022)

[SGA] Théorie des topos et cohomologie étale des schémas. Tome 2, Lecture Notes in Mathematics, Vol. 270, Springer-Verlag, Berlin, 1972. Séminaire de Géométrie Algébrique du 
Bois-Marie 1963-1964 (SGA 4), Dirigé par M. Artin, A. Grothendieck et J. L. Verdier. Avec la collaboration de N. Bourbaki, P. Deligne et B. Saint-Donat.

[Sp] David I. Spivak, Derived smooth manifolds, Duke Math. J. 153 (2010), no. 1, 55-128, DOI 10.1215/00127094-2010-021. MR2641940(2012a:57043)

Department of Mathematics, University of Texas, Austin, Texas 78712

E-mail address: dafr@math.utexas.edu

Department of Mathematics, Harvard University, Cambridge, Massachusetts 02138

E-mail address: mjh@math.harvard.edu 